\title{
Engineering precision nanoparticles for drug delivery
}

Michael J. Mitchell $\mathbb{1}^{1,2,3,4,5 凶}{ }^{\text {, Margaret M. Billingsley }}$, Rebecca M. Haley $\mathbb{1}^{1}$, Marissa E. Wechsler ${ }^{6}$. Nicholas A. Peppas ${ }^{6,7,8,9,10 凶}$ and Robert Langer(iD ${ }^{11 凶}$

Abstract | In recent years, the development of nanoparticles has expanded into a broad range of clinical applications. Nanoparticles have been developed to overcome the limitations of free therapeutics and navigate biological barriers - systemic, microenvironmental and cellular that are heterogeneous across patient populations and diseases. Overcoming this patient heterogeneity has also been accomplished through precision therapeutics, in which personalized interventions have enhanced therapeutic efficacy. However, nanoparticle development continues to focus on optimizing delivery platforms with a one-size-fits-all solution. As lipid-based, polymeric and inorganic nanoparticles are engineered in increasingly specified ways, they can begin to be optimized for drug delivery in a more personalized manner, entering the era of precision medicine. In this Review, we discuss advanced nanoparticle designs utilized in both non-personalized and precision applications that could be applied to improve precision therapies. We focus on advances in nanoparticle design that overcome heterogeneous barriers to delivery, arguing that intelligent nanoparticle design can improve efficacy in general delivery applications while enabling tailored designs for precision applications, thereby ultimately improving patient outcome overall.

Engineered nanomaterials hold significant promise to improve disease diagnosis and treatment specificity. Nanotechnology could help overcome the limitations of conventional delivery - from large-scale issues such as biodistribution to smaller-scale barriers such as intracellular trafficking - through cell-specific targeting, molecular transport to specific organelles and other approaches. To facilitate the realization and clinical translation of these promising nano-enabled technologies, the US National Science and Technology Council (NSTC) launched the National Nanotechnology Initiative (NNI) in 2000 and outlined well-defined initiatives and grand challenges for the field ${ }^{1}$. These initiatives have supported the recent efforts to investigate and improve nanotechnology, of which nanoparticles (NPs) constitute a significant portion of reported research and advancement.

NPs have the potential to improve the stability and solubility of encapsulated cargos, promote transport across membranes and prolong circulation times to increase safety and efficacy ${ }^{2,3}$. For these reasons, NP research has been widespread, generating promising results in vitro and in small animal models ${ }^{4}$. However, despite this extensive research motivated by the NNI, the number of nanomedicines available to patients is drastically below projections for the field, partially because of a translational gap between animal and human studies ${ }^{4,5}$. This gap comes from a lack of understanding of the differences in physiology and pathology between animal model species and humans, specifically how these differences influence the behaviour and functionality of nanomedicines in the body ${ }^{6}$. The differences across species are not the only factor that limits clinical translation. Heterogeneity amongst patients can also limit the success of nanomedicines, and there is currently only limited research on the interactions between nanomedicines and in stratified patient populations. Thus, of the nanomedicines that are approved, few are recommended as first-line treatment options, and many show

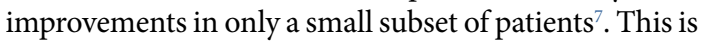
due, in part, to the underexplored heterogeneity both in the biological underpinnings of diseases and amongst patients, which alters NP efficacy because the growth, structure and physiology of diseased tissue alter NP distribution and functionality.

Many early NP iterations were unable to overcome these biological barriers to delivery, but more recent NP designs have utilized advancements in controlled synthesis strategies to incorporate complex architectures, bio-responsive moieties and targeting agents to enhance delivery ${ }^{8-12}$. These NPs can therefore be utilized as more complex systems - including in nanocarrier-mediated 
Reticuloendothelial system A system of specialized cells that clear foreign bodies from blood circulation.

PEGylated

Having polyethylene glycol (PEG) polymer chains attached, typically to molecules or macrostructures. combination therapies - to alter multiple pathways, maximize the therapeutic efficacy against specific macromolecules, target particular phases of the cell cycle or overcome mechanisms of drug resistance.

This new focus on generating NPs to overcome biological barriers specific to patient subsets or disease states can be attributed, in part, to the increasing prevalence of precision, or personalized, medicine and the creation of the Precision Medicine Initiative (PMI) in 2015 (REF. ${ }^{13}$ ). The goal of precision medicine is to utilize patient information - such as genetic profile, environmental exposures or comorbidities - to develop an individualized treatment plan. The use of precision minimizes the impact of patient heterogeneity and allows for more accurate patient stratification, improved drug specificity and optimized dosing or combinatorial strategies. However, precision therapies are subject to the same biological barriers to delivery as other medicines, which limits their clinical potential. As such, new NP designs, informed by patient data and engineered to overcome particular barriers in a stratified patient population, could greatly improve the delivery of and response to precision medicine therapies.

This Review focuses on advances in nanomedicine that could facilitate clinical translation of precision medicines and improve patient-specific therapeutic responses, with an emphasis on leveraging biomaterials and biomedical engineering innovations to overcome biological barriers and patient heterogeneity. The Review presents the progress made towards goals set forth by the NNI and the PMI to improve disease treatment for the individual. Although NPs have been used successfully in precision diagnostic applications, this Review focuses on the delivery of precision medicine therapeutics, as we believe that these medicines will greatly influence precision NPs in the future. Further, we discuss the biological barriers that have limited the widespread success of NP applications and critically review rational NP designs that have aimed to overcome these obstacles. The distribution and delivery trends from decades of NP research are also covered, as the impact of NP characteristics on therapeutic responses are explored. These emerging topics - as well as advances in engineering NPs for

\footnotetext{
Author addresses

${ }^{1}$ Department of Bioengineering, University of Pennsylvania, Philadelphia, PA, USA

${ }^{2}$ Abramson Cancer Center, Perelman School of Medicine, University of Pennsylvania, Philadelphia, PA, USA.

${ }^{3}$ Institute for Immunology, Perelman School of Medicine, University of Pennsylvania, Philadelphia, PA, USA.

${ }^{4}$ Cardiovascular Institute, Perelman School of Medicine, University of Pennsylvania, Philadelphia, PA, USA.

${ }^{5}$ Institute for Regenerative Medicine, Perelman School of Medicine, University of Pennsylvania, Philadelphia, PA, USA.

${ }^{6}$ Department of Biomedical Engineering, The University of Texas at Austin, Austin, TX, USA ${ }^{7}$ Department of Chemical Engineering, The University of Texas at Austin, Austin, TX, USA. ${ }^{8}$ Department of Pediatrics, The University of Texas at Austin, Austin, TX, USA.

${ }^{9}$ Department of Surgery and Perioperative Care, The University of Texas at Austin, Austin, TX, USA.

${ }^{10}$ Department of Molecular Pharmaceutics and Drug Delivery, The University of Texas at Austin, Austin, TX, USA.

${ }^{11}$ Department of Chemical Engineering and Koch Institute for Integrative Cancer

Research, Massachusetts Institute of Technology, Cambridge, MA, USA.
}

specific applications - are of particular importance as new opportunities arise for the clinical translation of NP-based precision therapies in cancer medicine, immunotherapy and in vivo gene editing (FIG. 1).

\section{NP classes \\ Lipid-based NPs}

Lipid-based NPs include various subset structures but are most typically spherical platforms comprising at least one lipid bilayer surrounding at least one internal aqueous compartment (FIG. 2). As a delivery system, lipid-based NPs offer many advantages including formulation simplicity, self-assembly, biocompatibility, high bioavailability, ability to carry large payloads and a range of physicochemical properties that can be controlled to modulate their biological characteristics ${ }^{14,15}$. For these reasons, lipid-based NPs are the most common class of FDA-approved nanomedicines ${ }^{7,16}$ (TABLE 1).

For liposomes - one of the subsets of lipid-based NPs that has the most members - the NPs are typically composed of phospholipids, which can form unilamellar and multilamellar vesicular structures. This allows the liposome to carry and deliver hydrophilic, hydrophobic and lipophilic drugs, and they can even entrap hydrophilic and lipophilic compounds in the same system, thereby expanding their use ${ }^{17}$. Their in vitro and in vivo stability are altered by NP size, surface charge, lipid composition, number of lamellae and surface modifications (with ligands or polymers), which can be altered during synthesis ${ }^{15,18}$. Because they can be rapidly taken up by the reticuloendothelial system, liposomes often include surface modifications to extend their circulation and enhance delivery, which has enabled their clinical use ${ }^{14,19}$.

Another notable subset of lipid-based NPs is commonly referred to as lipid nanoparticles (LNPs) liposome-like structures widely used for the delivery of nucleic acids. They differ from traditional liposomes primarily because they form micellar structures within the particle core, a morphology that can be altered based on formulation and synthesis parameters ${ }^{20}$. LNPs are typically composed of four major components: cationic or ionizable lipids that complex with negatively charged genetic material and aid endosomal escape, phospholipids for particle structure, cholesterol for stability and membrane fusion, and PEGylated lipids to improve stability and circulation ${ }^{21,22}$. The efficacy of their nucleic acid delivery along with their simple synthesis, small size and serum stability have made LNPs particularly important in personalized genetic therapy applications ${ }^{12,23}$. Ionizable LNPs are an ideal platform for the delivery of these nucleic acid therapies as they have a near-neutral charge at physiological $\mathrm{pH}$ but become charged in acidic endosomal compartments, promoting endosomal escape for intracellular delivery ${ }^{24,25}$. However, despite these advantages, LNP systems can still be limited by low drug loading and biodistribution that results in high uptake to the liver and spleen ${ }^{16}$.

\section{Polymeric NPs}

Polymeric NPs can be synthesized from natural or synthetic materials, as well as monomers or preformed polymers - allowing for a wide variety of possible 


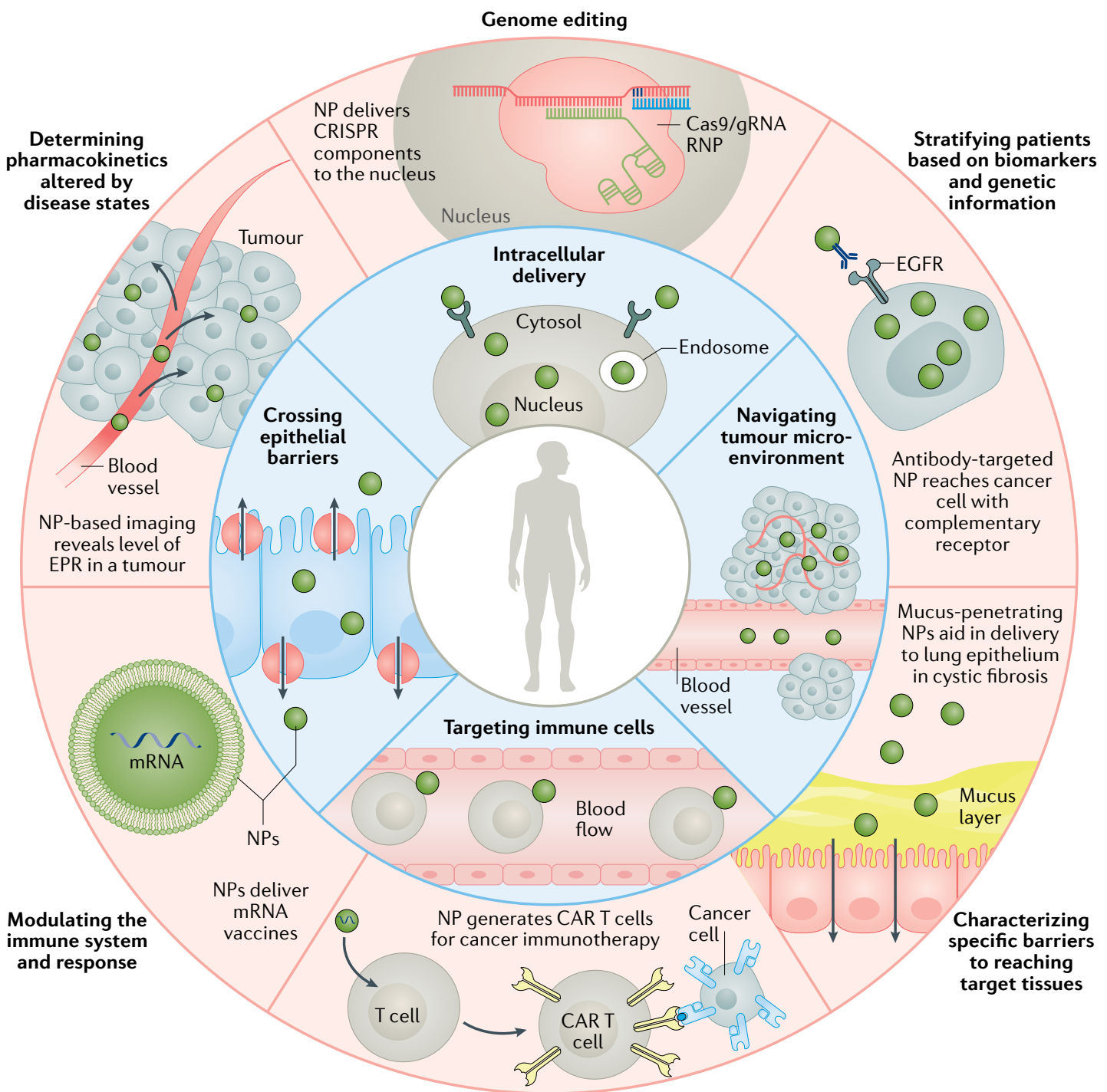

Creating autologous cell therapies

Fig. 1 | Biological barriers to precision medicine applications. Overview highlighting some of the biological barriers that nanoparticles (NPs) can overcome (inner ring) and precision medicine applications that may benefit from NPs (outer ring). As explored in this Review, intelligent NP designs that improve delivery have the potential to enhance the performance of precision medicines and, thus, accelerate their clinical translation. CAR, chimeric antigen receptor; EGFR, epidermal growth factor receptor; EPR, enhanced permeation and retention; gRNA, guide RNA; RNP, ribonucleoprotein.

structures and characteristics (FIG. 2). They can be formulated to enable precise control of multiple NP features and are generally good delivery vehicles because they are biocompatible and have simple formulation parameters. Polymeric NPs are synthesized using various techniques such as emulsification (solvent displacement or diffusion $)^{26}$, nanoprecipitation ${ }^{27,28}$, ionic gelation ${ }^{29}$ and microfluidics ${ }^{30}$, which all result in different final products. Polymeric NPs also have variable drug delivery capabilities. Therapeutics can be encapsulated within the NP core, entrapped in the polymer matrix, chemically conjugated to the polymer or bound to the NP surface. This enables delivery of various payloads including hydrophobic and hydrophilic compounds, as well as cargos with different molecular weights such as small molecules, biological macromolecules, proteins and vaccines ${ }^{30-35}$, making polymeric NPs ideal for co-delivery applications ${ }^{36}$. By modulating properties such as composition, stability, responsivity and surface charge, the loading efficacies and release kinetics of these therapeutics can be precisely controlled ${ }^{37,38}$.

The most common forms of polymeric NPs are nanocapsules (cavities surrounded by a polymeric membrane or shell) and nanospheres (solid matrix systems). Within these two large categories, NPs are divided further into shapes such as polymersomes, micelles and dendrimers. Polymersomes are artificial vesicles, with membranes made using amphiphilic block copolymers. They are comparable to liposomes, and are often locally responsive, but are reported to have improved stability and cargo-retention efficiency ${ }^{39}$, making them effective vehicles for the delivery of therapeutics to the cytosol ${ }^{40,41}$. Some polymers which are commonly copolymerized for these applications include poly(ethylene glycol) 
Polymeric

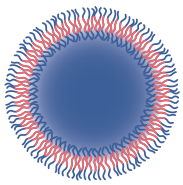

Polymersome

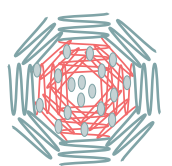

Polymer micelle

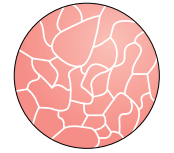

Dendrimer

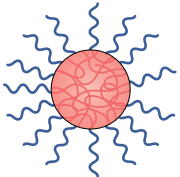

Nanosphere

- Precise control of particle characteristics

- Payload flexibility for hydrophilic and hydrophobic cargo

- Easy surface modification

- Possibility for aggregation

and toxicity
Inorganic

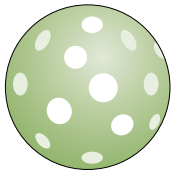

Silica NP

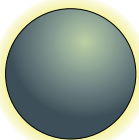

Iron oxide NP

- Unique electrical, magnetic and optical properties

- Variability in size, structure

and geometry

- Well suited for theranostic

applications

- Toxicity and solubility limitations
Lipid-based
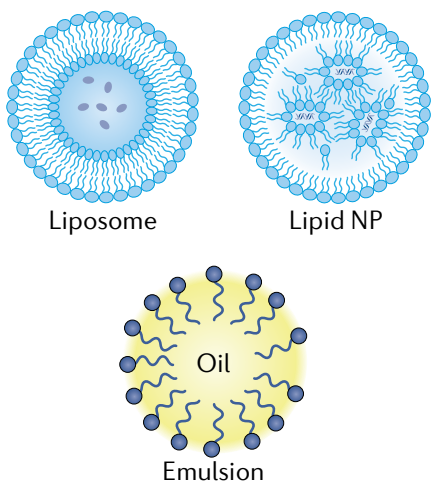

- Formulation simplicity with a range of physicochemical properties

- High bioavailability

- Payload flexibility

- Low encapsulation

efficiency

Fig. 2 | Classes of NPs. Each class of nanoparticle (NP) features multiple subclasses, with some of the most common highlighted here. Each class has numerous broad advantages and disadvantages regarding cargo, delivery and patient response.

(PEG) and poly(dimethylsiloxane) (PDMS). Polymeric micelles, which are also typically responsive block copolymers, self-assemble to form nanospheres with a hydrophilic core and a hydrophobic coating: this serves to protect aqueous drug cargo and improve circulation times. Polymeric micelles can load various therapeutic types - from small molecules to proteins ${ }^{35}$ - and have been used for the delivery of cancer therapeutics in clinical trials ${ }^{42}$.

Dendrimers are hyperbranched polymers with complex three-dimensional architectures for which the mass, size, shape and surface chemistry can be highly controlled. Active functional groups present on the exterior of dendrimers enable conjugation of biomolecules or contrast agents to the surface while drugs can be loaded in the interior. Dendrimers can hold many types of cargo, but are most commonly investigated for the delivery of nucleic acids and small molecules ${ }^{43,44}$. For these applications, charged polymers such as poly(ethylenimine) (PEI) and poly(amidoamine) (PAMAM) are commonly used. Several dendrimer-based products are currently in clinical trials as theranostic agents, transfection agents, topical gels and contrast agents ${ }^{44-46}$. Charged polymers can be used to form non-dendrimer NPs as well. Polyelectrolytes are one such example: these polymers have a repeating electrolyte group, giving them charge that varies with $\mathrm{pH}$. Polyelectrolytes have been incorporated in numerous NP formulations to improve properties such as bioavailability ${ }^{47}$ and mucosal transport ${ }^{48}$. They are also inherently responsive, and can be useful for intracellular delivery.

Overall, polymeric NPs are ideal candidates for drug delivery because they are biodegradable, water soluble, biocompatible, biomimetic and stable during storage. Their surfaces can be easily modified for additional targeting ${ }^{49}-$ allowing them to deliver drugs, proteins and genetic material to targeted tissues, which makes them useful in cancer medicine, gene therapy and diagnostics. However, disadvantages of polymeric NPs include an increased risk of particle aggregation and toxicity. Only a small number of polymeric nanomedicines are currently FDA approved and used in the clinic (TABLE 1), but polymeric nanocarriers are currently undergoing testing in numerous clinical trials ${ }^{7}$.

\section{Inorganic NPs}

Inorganic materials such as gold, iron and silica have been used to synthesize nanostructured materials for various drug delivery and imaging applications (FIG. 2). These inorganic NPs are precisely formulated and can be engineered to have a wide variety of sizes, structures and geometries. Gold NPs (AuNPs), which are the most well studied, are used in various forms such as nanospheres, nanorods, nanostars, nanoshells and nanocages $^{50}$. Additionally, inorganic NPs have unique physical, electrical, magnetic and optical properties, due to the properties of the base material itself. For example, AuNPs possess free electrons at their surface that continually oscillate at a frequency dependent on their size and shape, giving them photothermal properties ${ }^{51}$. AuNPs are also easily functionalized, granting them additional properties and delivery capabilities ${ }^{50}$.

Iron oxide is another commonly researched material for inorganic NP synthesis, and iron oxide NPs make up the majority of FDA-approved inorganic nanomedicines ${ }^{52}$ (TABLE 1). Magnetic iron oxide NPs - composed of magnetite $\left(\mathrm{Fe}_{3} \mathrm{O}_{4}\right)$ or maghemite $\left(\mathrm{Fe}_{2} \mathrm{O}_{3}\right)$ - possess superparamagnetic properties at certain sizes and have shown success as contrast agents, drug delivery vehicles and thermal-based therapeutics ${ }^{53}$. Other common inorganic NPs include calcium phosphate and mesoporous silica NPs, which have both been used successfully for gene and drug delivery ${ }^{5,55}$. Quantum dots - typically made of semiconducting materials such as silicon - are unique 
NPs used primarily in in vitro imaging applications, but they show promise for in vivo diagnostics ${ }^{56,57}$.

Due to their magnetic, radioactive or plasmonic properties, inorganic NPs are uniquely qualified for applications such as diagnostics, imaging and photothermal therapies. Most have good biocompatibility and stability, and fill niche applications that require properties unattainable by organic materials. However, they are limited in their clinical application by low solubility and toxicity concerns, especially in formulations using heavy metals ${ }^{53,58}$.

\section{NPs in precision medicine}

Precision medicine pushes for the development of patient-specific treatments in a clinical setting, to overcome the many limitations of traditional one-size-fitsall approaches and improve therapeutic outcomes ${ }^{59}$. In oncology, patient stratification through biomarkers and companion diagnostics has become the norm for drug development, as most cancer nanomedicines fail to produce positive results in unstratified studies ${ }^{60}$.
Even though patient stratification has been essential in the clinical development of several precision medicines for cancer, NP-based clinical trials are currently conducted in unstratified patient populations ${ }^{61}$. However, this will likely change in the near future, as the importance of stratification becomes more apparent and NPs begin to be developed with specific patient populations in mind. The progression of NPs through clinical trials may similarly be hastened by the incorporation of stratified patient populations, as these populations will likely respond more uniformly to treatment. Furthermore, NPs are particularly well placed to broaden the potential patient populations that qualify for precision medicine therapies by neutralizing factors, such as comorbidities or heterogeneous biological barriers, that may have made patients previously unqualified. As NPs overcome many of the current limitations to delivery - potentially improving the potency and therapeutic efficacy of precision medicines - they may allow more patients to qualify for clinical trials and benefit from individualized therapies.

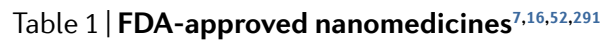

\begin{tabular}{|c|c|c|c|}
\hline Drug & Company & Application & $\begin{array}{l}\text { Date of first } \\
\text { approval }\end{array}$ \\
\hline \multicolumn{4}{|l|}{ Lipid-based } \\
\hline Doxil & Janssen & Kaposi’s sarcoma, ovarian cancer, multiple myeloma & 1995 \\
\hline DaunoXome & Galen & Kaposi's sarcoma & 1996 \\
\hline AmBisome & Gilead Sciences & Fungal/protozoal infections & 1997 \\
\hline Visudyne & Bausch and Lomb & $\begin{array}{l}\text { Wet age-related macular degeneration, myopia, } \\
\text { ocular histoplasmosis }\end{array}$ & 2000 \\
\hline Marqibo & Acrotech Biopharma & Acute lymphoblastic leukaemia & 2012 \\
\hline Onivyde & Ipsen & Metastatic pancreatic cancer & 2015 \\
\hline Vyxeos & Jazz Pharmaceuticals & Acute myeloid leukaemia & 2017 \\
\hline Onpattro & Alnylam Pharmaceuticals & Transthyretin-mediated amyloidosis & 2018 \\
\hline \multicolumn{4}{|c|}{ Polymer-based } \\
\hline Oncaspar & Servier Pharmaceuticals & Acute lymphoblastic leukaemia & 1994 \\
\hline Copaxone & Teva & Multiple sclerosis & 1996 \\
\hline Peglntron & Merck & Hepatitis C infection & 2001 \\
\hline Eligard & Tolmar & Prostate cancer & 2002 \\
\hline Neulasta & Amgen & Neutropenia, chemotherapy induced & 2002 \\
\hline Abraxane & Celgene & $\begin{array}{l}\text { Lung cancer, metastatic breast cancer, metastatic } \\
\text { pancreatic cancer }\end{array}$ & 2005 \\
\hline Cimiza & UCB & $\begin{array}{l}\text { Crohn's disease, rheumatoid arthritis, psoriatic } \\
\text { arthritis, ankylosing spondylitis }\end{array}$ & 2008 \\
\hline Plegridy & Biogen & Multiple sclerosis & 2014 \\
\hline ADYNOVATE & Takeda & Haemophilia & 2015 \\
\hline \multicolumn{4}{|l|}{ Inorganic } \\
\hline INFeD & Allergan & Iron-deficient anaemia & 1992 \\
\hline DexFerrum & American Regent & Iron-deficient anaemia & 1996 \\
\hline Ferrlecit & Sanofi & Iron deficiency in chronic kidney disease & 1999 \\
\hline Venofer & American Regent & Iron deficiency in chronic kidney disease & 2000 \\
\hline Feraheme & AMAG & Iron deficiency in chronic kidney disease & 2009 \\
\hline Injectafer & American Regent & Iron-deficient anaemia & 2013 \\
\hline
\end{tabular}


Since the launch of the PMI in 2015, several applications have incorporated nanomaterials in precision medicine $e^{59}$. For example, a blood test for the early detection of pancreatic cancer analyses the personalized biomolecular corona that adsorbs onto graphene oxide nanoflakes ${ }^{62}$. The unique property of graphene oxide, which binds low amounts of albumin, allows strong adsorption of proteins that are present in the plasma at low levels ${ }^{62}$. Other studies use magnetic $\mathrm{NPs}^{63}$ or AuNPs ${ }^{64,65}$, which are simple to use, in biomarker detection assays, thereby saving time and money if compared with existing methods that require substantial sample processing. In addition to diagnostic screening, some therapeutic applications of NPs aim to remodel the tumour microenvironment to promote particle accumulation and penetration, and thus increase drug efficacy, and/or to sensitize tumours to a particular therapy ${ }^{66-68}$. For example, tumour-associated endothelial cells can be manipulated by a NP-delivered microRNA, which alters the tumour vasculature and thereby sensitizes the tumour to traditional cancer therapies ${ }^{67}$. Similarly, bio-inspired lipoproteins have been used to remodel tumours, and can improve NP accessibility to cancer cells 27 -fold ${ }^{66}$. The usage of photothermal NPs can improve the infiltration and activity of chimeric antigen receptor (CAR) T cells against solid tumours ${ }^{69}$. NPs can also be used to modulate immune activation or suppression to sensitize cancer cells to therapeutics, helping to homogenize these currently heterogeneous environments in an attempt to increase the number of patients who respond to or qualify for precision treatments ${ }^{40,70}$.

In summary, combining NPs and precision medicine has the potential to advance both fields. Because NPs are currently screened in unstratified patient populations, the introduction of NPs developed for specific patient populations could allow for the accelerated clinical translation of numerous nanomaterials. Conversely, the success of precision medicine relies on strictly stratified patient populations, and the use of NPs to improve delivery across heterogeneous biological barriers could increase the efficacy of precision medicines, allowing for more patients to be included in the stratified population, as well as increasing the likelihood of successful translation to the clinic. Advances in genome sequencing and biomarker detection allow for the appropriate selection of cargo for the treatment of patient-specific diseases. Although it is not the focus of this Review, there are several diagnostic applications that may be improved by NP technologies. Development of nanobiomaterials for precision medicine is a highly customizable process. This careful design approach enables adjustments of the therapeutics' pharmacokinetics to match requirements for solubility, administration or biodistribution and has seen success in research settings (TABLE 2).

Complement system A group of distinct plasma proteins that induce inflammation and aid in the clearance of foreign bodies or damaged cells by enhancing antibody and phagocytic cell activity. are often altered in disease states and can be even more difficult to overcome with a generalized, one-size-fits-all approach $^{3,72-74}$. Furthermore, these changes in biological barriers vary not just across diseases but also on a patient-to-patient basis, and they can occur at the systemic, microenvironmental and cellular levels, making them hard to isolate and characterize broadly. Understanding the biological barriers faced both generally and on a patient-specific level allows for the design of optimally engineered NP platforms. In this section, we discuss strategies used by NPs to overcome biological barriers on the systemic, local and cellular scale (FIG. 3).

\section{Systemic delivery and biodistribution}

The biological barriers that NPs encounter depend on the route of administration as well as the patient's disease type and progression ${ }^{3}$. Although local delivery methods may allow NPs to circumvent some of the obstacles faced by systemic delivery, they often involve more invasive procedures and complex techniques that present other limitations. Furthermore, local delivery may only be useful in diseases where the pathology is restricted to known, accessible sites - such as certain solid cancers or traumatic injuries - so systemic administration is more common in NP applications ${ }^{75}$. Thus, this section explores the most prominent barriers to delivery faced by systemic administration.

Circulation, stability and clearance. While in circulation, factors such as excretion, blood flow, coronas and phagocytic cells can reduce NP stability and delivery (FIG. 3). The specific effects of each of these environmental factors is dependent upon the physiochemical properties of the NP platform, which has led to general design principles aimed to manipulate these characteristics to achieve favourable outcomes. In size, for example, NPs with a diameter less than $10 \mathrm{~nm}$ have generally been shown to be rapidly eliminated by the kidneys, whereas NPs larger than $200 \mathrm{~nm}$ risk activating the complement system, if not otherwise engineered ${ }^{76}$. Furthermore, to avoid rapid excretion based on surface properties, many NP formulations incorporate PEG as a stealth coating. PEGylation improves the circulation time by altering the NP size and solubility while shielding the NP surface from enzymes and antibodies that may induce degradation, secretion and clearance, but this physical barrier does not completely prevent recognition by macrophages or other cells of the immune system. Additionally, exposure to PEG results in the production of anti-PEG antibodies that, when present in high concentrations, can induce the rapid clearance of PEGylated NPs ${ }^{77,78}$. Clinical studies have also shown that these anti-PEG antibodies can be present in humans who have been exposed to PEG through means other than PEGylated medicines, indicating that even the first dose of PEGylated NPs would not necessarily circulate for long in all patients ${ }^{79,80}$.

Another option for stealth is platelet membrane cloaking, which reduces cell uptake and complement activation ${ }^{81}$. Although this cloaking avoids the macrophage-based immune issues associated with PEGylation, the NPs may still be recognized by other 
Table 2 | NPs investigated for precision medicine as a therapeutic or diagnostic device

\begin{tabular}{|c|c|c|c|}
\hline NP type & $\begin{array}{l}\text { Primary design } \\
\text { advancements }\end{array}$ & $\begin{array}{l}\text { Therapeutic cargo or target } \\
\text { for detection }\end{array}$ & Indication or application \\
\hline \multicolumn{4}{|l|}{ Therapies } \\
\hline \multirow[t]{8}{*}{ LNPs } & Charge & mRNA & Immunotherapies for cancer or autoimmune diseases 227 \\
\hline & \multirow[t]{3}{*}{ Responsivity } & $\begin{array}{l}\text { Small molecule, photothermal } \\
\text { agent }\end{array}$ & Metastatic breast cancer ${ }^{66}$ \\
\hline & & mRNA & $\begin{array}{l}\left.\text { Retinal disorders }{ }^{25} \text {; melanoma, human papillomavirus E7 (REF. }{ }^{174}\right) \text {; } \\
\text { anaemic disorders }{ }^{175} \text {; acute lymphoblastic leukaemia }{ }^{258}\end{array}$ \\
\hline & & Cyclic dinucleotide & Lung metastasis of melanoma and breast cancer ${ }^{248}$ \\
\hline & Responsivity and charge & mRNA, protein & Diseases of the lung and spleen ${ }^{12}$ \\
\hline & Surface modification & siRNA & Pulmonary diseases ${ }^{176}$ \\
\hline & $\begin{array}{l}\text { Surface modification and } \\
\text { responsivity }\end{array}$ & pDNA & Osteoporosis $^{24}$ \\
\hline & $\begin{array}{l}\text { Surface modification and } \\
\text { charge }\end{array}$ & siRNA & Hepatocellular carcinoma ${ }^{99}$ \\
\hline \multirow[t]{20}{*}{ Polymer NPs } & Shape & NA; observed distribution & Neurological diseases with inflammation ${ }^{91}$; cervical cancer ${ }^{163}$ \\
\hline & \multirow[t]{9}{*}{ Responsivity } & Small molecule & Non-small cell lung cancer ${ }^{212}$; lung carcinoma ${ }^{219}$ \\
\hline & & Protein & Diabetes $^{35,38}$ \\
\hline & & Protein, small molecule & Breast cancer ${ }^{208}$; immunotherapies for cancer ${ }^{180}$ \\
\hline & & Protein, gRNA & Monogenetic diseases of the eye $\mathrm{e}^{173}$ \\
\hline & & Protein, ssDNA & Vaccination (influenza A H1N1, vaccinia) ${ }^{32}$ \\
\hline & & Anti-sense RNA & Mitochondrial disorders ${ }^{183}$ \\
\hline & & siRNA & Pancreatic adenocarcinomas ${ }^{33} ;$ glioblastoma ${ }^{200}$ \\
\hline & & siRNA, small molecule & Cancer $^{181}$ \\
\hline & & Cyclic dinucleotide & Metastatic melanoma $^{40}$ \\
\hline & \multirow[t]{5}{*}{ Surface modification } & Small molecule & $\begin{array}{l}\text { Head and neck cancer }{ }^{196} ; \text { TNBC }^{49} ; \text { systemic lupus erythematosus } \\
\text { myocardial ischaemia reperfusion injury }\end{array}$ \\
\hline & & mRNA & Ovarian cancer, melanoma, glioblastoma ${ }^{238} ;$ liver disease $^{100}$ \\
\hline & & mRNA, DNA & Cystic fibrosis ${ }^{283}$ \\
\hline & & Dyes & Glioblastoma ${ }^{154}$ \\
\hline & & NA; observed distribution & Osteoarthritis $^{26} ;$ nuclear delivery ${ }^{41}$ \\
\hline & \multirow[t]{5}{*}{$\begin{array}{l}\text { Surface modification and } \\
\text { responsivity }\end{array}$} & Small molecule & $\begin{array}{l}\text { Ovarian cancer }{ }^{123} \text {; breast cancer }{ }^{198} ; \text { hepatocellular carcinoma }{ }^{147} \text {; } \\
\text { cancer }^{84}\end{array}$ \\
\hline & & $\begin{array}{l}\text { Small molecule, peptide, } \\
\text { protein }\end{array}$ & Colorectal cancer ${ }^{11}$ \\
\hline & & siRNA, pDNA & Hepatocellular carcinoma ${ }^{54}$ \\
\hline & & Antibody, miRNA & Colorectal cancer ${ }^{126}$ \\
\hline & & Antibody, photosensitizer & Metastatic breast cancer ${ }^{205}$ \\
\hline \multirow[t]{10}{*}{ Inorganic NPs } & \multirow[t]{2}{*}{ Responsivity } & Small molecule, imaging agent & Breast cancer ${ }^{203}$ \\
\hline & & $\begin{array}{l}\text { Neoantigen, adjuvant, } \\
\text { photosensitizer }\end{array}$ & Colon carcinoma, melanoma ${ }^{55}$ \\
\hline & \multirow[t]{4}{*}{ Surface modification } & Photosensitizer & Oral squamous cell carcinoma ${ }^{102}$ \\
\hline & & siRNA & Breast cancer ${ }^{122}$ \\
\hline & & miRNA & TNBC $^{201}$ \\
\hline & & NA; observed distribution & Neurological disorders ${ }^{130}$, glioblastoma $^{156}$ \\
\hline & \multirow{2}{*}{$\begin{array}{l}\text { Surface modification and } \\
\text { responsivity }\end{array}$} & Protein, antibody & Mitochondrial dysfunctions ${ }^{182}$ \\
\hline & & Small molecule & TNBC $^{239}$ \\
\hline & $\begin{array}{l}\text { Surface modification and } \\
\text { size }\end{array}$ & $\begin{array}{l}\text { NPs for magnetic } \\
\text { hyperthermia }\end{array}$ & Breast cancer ${ }^{125}$ \\
\hline & $\begin{array}{l}\text { Surface modification and } \\
\text { shape }\end{array}$ & Small molecule & Non-small cell lung carcinoma ${ }^{165}$ \\
\hline
\end{tabular}


Table 2 (cont.) | NPs investigated for precision medicine as a therapeutic or diagnostic device

\begin{tabular}{|c|c|c|c|}
\hline NP type & $\begin{array}{l}\text { Primary design } \\
\text { advancements }\end{array}$ & $\begin{array}{l}\text { Therapeutic cargo or target } \\
\text { for detection }\end{array}$ & Indication or application \\
\hline \multicolumn{4}{|l|}{ Diagnostics } \\
\hline LNPs & Surface modification & $\begin{array}{l}{ }^{64} \mathrm{Cu} \text { and small molecule; to } \\
\text { detect cells }\end{array}$ & Metastatic breast cancer ${ }^{152}$ \\
\hline Polymer NPs & Surface modification & $\begin{array}{l}\text { Fluorescent dyes or }{ }^{64} \mathrm{Cu} \text {; to } \\
\text { detect cells }\end{array}$ & Epidermoid cancer 121 \\
\hline \multirow{4}{*}{ Inorganic NPs } & \multirow[t]{4}{*}{ Surface modification } & $\beta$-Amyloid peptide & Alzheimer disease ${ }^{57}$ \\
\hline & & Heat shock protein & Tuberculosis ${ }^{293}$ \\
\hline & & Blood iron level & Anaemia ${ }^{294}$ \\
\hline & & Thrombin & Cancer $^{64}$ \\
\hline
\end{tabular}

gRNA, guide RNA; LNP, lipid nanoparticle; miRNA, micro-RNA; NA, not applicable; NP, nanoparticle; pDNA, plasmid DNA; siRNA, small interfering RNA; sSDNA, single-stranded DNA; TNBC, triple-negative breast cancer.

cell populations ${ }^{82}$. However, these platelet-based cell interactions can also help with targeting: platelet membrane-cloaked NPs feature the ligands present on native platelets - including mediators of adhesion to von Willebrand factor and collagen - allowing the wrapped NPs to target injury sites and accumulate around activated platelets ${ }^{83}$.

Surface modifications and cloaking techniques allow NPs to avoid the recognition and clearance systems that may lead to rapid NP degradation and instability, and there are also numerous NP design strategies that specifically focus on improving stability. NP stability is greatly affected by how its composition material interacts with the environment, and lipid-based and polymer-based NPs are the most susceptible to instability and aggregation both in circulation and in storage. Thus, to improve the robustness of these softer NPs, excipients such as helper lipids, cholesterol and PEGylated lipids ${ }^{18,21}$ can be formulated with lipid-based NPs to increase their stability, whereas polymer NPs may utilize cross-linking techniques ${ }^{84,85}$. For storage and transport, many NPs are lyophilized to improve stability, although this does not affect the NP stability once administered ${ }^{21}$. However, as NP designs aim to increase stability, the balance between stabilization and effective intracellular delivery which typically requires carrier degradation - must be considered.

In the bloodstream, NPs experience varying flow rates that induce shear stress and may damage the platforms or their cargo and prevent extravasation ${ }^{86,87}$. These fluid forces can strip NPs of their surface coatings and can prevent NPs from localizing to vessel walls to extravasate - either transcellularly or paracellularly to reach target tissues ${ }^{3,86-90}$. Larger (microscale) particles have a higher probability of localizing to the vessel walls, and non-spherical particles show better margination ${ }^{89}$. Specifically, ellipsoids, discoid shapes and nanorods with higher aspect ratios localize to blood vessels better than spheres $\mathrm{do}^{89,91,92}$. This is caused by flow-induced rolling in shapes with high ratios, which results in edge margination at a speed proportional to the NP aspect ratio ${ }^{3}$. Even after vessel localization, architecture-dependent drag force from blood flow may rip NPs from cell membranes if they lack sufficient binding affinity for endothelial cells ${ }^{88}$. Thus, the haemodynamics experienced by systemically administered NPs - which are often altered in vascular pathologies such as stenoses and hypertension ${ }^{93,94}$ - greatly influence NP distribution and delivery.

In addition to their interactions with vessel walls, circulating NPs come into contact with biomolecules and cells suspended in blood. The non-specific adherence of serum proteins and lipids forms a corona on the surface of $\mathrm{NPs}^{61,95,96}$. The composition of the corona depends on the biomolecules present in blood as well as the physicochemical characteristics of the NP surface, as this dictates the adsorption or desorption of proteins from biological fluids $s^{61,96}$. At times, the engineered surface properties meant to enhance NP targeting - such as conjugated ligands or modified surface charge - may encourage corona formation through charge-specific interactions $s^{97}$. Once formed, this corona will dictate the distribution of the NP, and can compromise stability of both the NP and its cargo ${ }^{61,96,98}$. Recent investigations have sought to determine how the specific corona biomolecules alter NP distribution and tissue-specific targeting ${ }^{99-101}$. For example, coronas containing apolipoprotein E (ApoE) act as targeting moieties for low-density lipoprotein receptors, which leads to NP delivery to hepatocytes and, in some instances, across the blood-brain barrier $(\mathrm{BBB})^{99,102-104}$. If the corona contains opsonin or ligands for pattern recognition receptors, it can cause rapid clearance of the NPs via contact with cells of the innate immune system ${ }^{98}$.

Clearance of NPs from the circulation can be influenced by their physicochemical properties, but often results from interactions with the mononuclear phagocytic system (MPS) or reticuloendothelial system ${ }^{3,97}$ (FIG. 3). These systems feature phagocytes (predominantly macrophages), monocytes and dendritic cells, which take up NPs and accumulate in the spleen and liver ${ }^{71,97,98}$. This clearance tends to happen more rapidly in stiffer $\mathrm{NPs}^{105,106}$. In terms of surface charge, cationic NPs are generally most rapidly cleared, followed by anionic NPs, whereas neutral and slightly negative NPs have the longest half-lives in circulation ${ }^{2,3}$. To minimize 
clearance, some NP designs implement surface modifications - such as PEG, 'self' peptides (including CD47) or cell membrane coatings - that aim to reduce these interactions with phagocytic cells of the MPS ${ }^{3,76}$.

In addition to clearance, interactions with the MPS can cause toxicity, as these cells trigger immune responses involving the secretion of tumour necrosis factors, interleukins and interferons that cause inflammation or tissue damage ${ }^{98,107}$. The type and magnitude of immune response to NPs is greatly affected by NP size, shape and surface properties. For example, in a mouse ovalbumin model, spherical NPs cause a T helper 1 cellbiased (cell-mediated) response, micrometre-length rods cause a T helper 2 cell-biased (humoral) response and spherical NPs induce a stronger immune response overall ${ }^{108}$. Furthermore, uptake by phagocytic cells has been related to the NP curvature and aspect ratio: triangular and rod-shaped NPs show more uptake than star-shaped or spherical NPs, and rod-shaped NPs induce more inflammation in macrophages ${ }^{109-111}$. Certain surface properties induce inflammation; some PEGylated NPs have caused severe allergic reactions or anaphylaxis in a small subset of patients in clinical trials $^{112,113}$. Although the steric effects of PEG on the surface of NPs typically prevent interactions with MPS cells, anti-PEG antibodies developed from previous PEG exposure undermine this stealth property and promote MPS interactions ${ }^{77,78}$. At high concentrations, anti-PEG antibodies most commonly cause rapid clearance, but they are also thought to contribute to these uncommon but severe allergic reactions. In all, these immune responses to NP architecture and surface modifications can induce inflammation and adverse reactions, which emphasizes the importance of tailoring NP design to minimize these risks ${ }^{61,82}$.

Barriers to biodistribution. Extravasation is the first step for a NP in circulation to reach the target tissue ${ }^{89,90}$. Extravasation can be altered by NP characteristics, including size: for example, small NPs generally cross capillary walls more easily than large NPs ${ }^{3,71,98}$ (FIG. 3). Thus, NPs tend to distribute across organs in a sizedependent manner, with the highest accumulation often in the liver and spleen ${ }^{3,76}$. However, size-dependent distribution can be altered by pathological environments such as the tumour vasculature, in which larger than normal intercellular gaps allow for larger NPs to exit the vessels $^{71}$. Overall, extravasation leads to non-specific distribution, which presents a translational challenge for applications that require specific localization ${ }^{3}$.

Optimizing the administration route can improve biodistribution. The means of administering any drug may alter its fate and efficacy in vivo, and numerous studies have explored how these routes impact the fate of NPs specifically ${ }^{114,115}$. For example, polymeric (poly(lactic-co-glycolic) acid (PLGA)) NPs that are intravenously injected accumulate primarily in the liver and spleen, whereas if these NPs are subcutaneously or
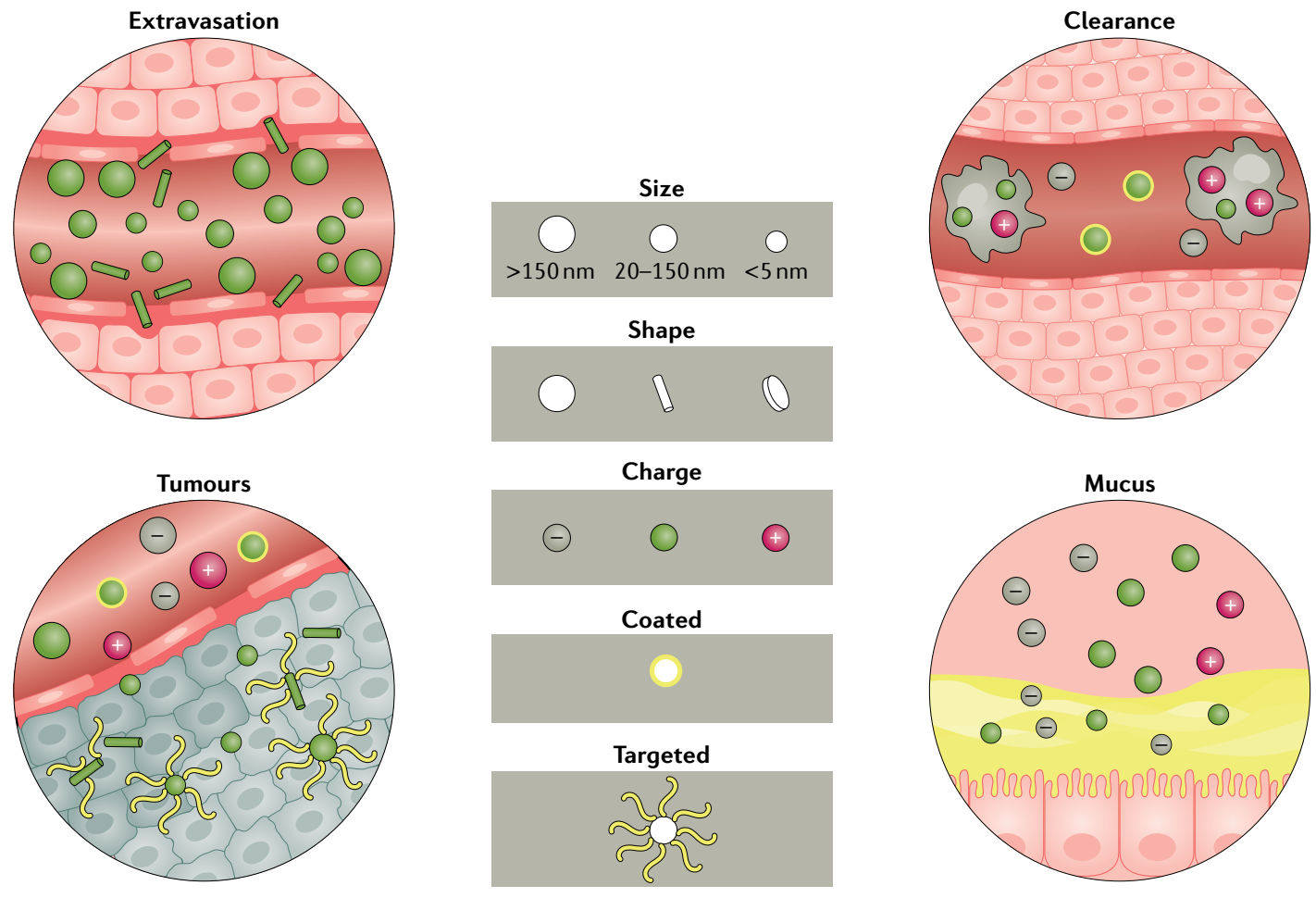

Fig. 3 | NP characteristics impact distribution. Factors such as size, shape, charge and surface coating determine what happens to nanoparticles (NPs) in the circulation, including clearance, and how the NPs interact with local barriers such as the tumour microenvironment or mucus layers. A few general trends are highlighted here: spherical and larger NPs marginate more easily during circulation, whereas rod-shaped NPs extravasate more readily (top left); and uncoated or positively charged NPs are cleared more quickly by macrophages (top right). In terms of local distribution, in general, rod-shaped, neutral and targeted NPs penetrate tumours more readily (bottom left) whereas positively charged, smaller and coated NPs more easily traverse mucosal barriers (bottom right). 
First-pass metabolism The metabolism of a drug within the liver and enterocytes before the drug reaches the systemic circulation. intranodally injected, they are more likely to accumulate in local lymph nodes ${ }^{116}$. These alternate administration routes enable NPs to reach the lymphatic system prior to systemic circulation, which could be beneficial in certain immunotherapeutic applications ${ }^{116,117}$. Another method for bypassing extravasation that has been increasingly explored for NP delivery is pulmonary administration, specifically NP inhalation. This route avoids exposure to the systemic circulation prior to lung delivery, thus avoiding hepatic first-pass metabolism and increasing the delivery of dendrimer-based NPs to the lung and lymph node as compared with intravenous delivery ${ }^{114}$. However, despite their improved delivery to lung tissue, inhaled NPs face the unique obstacles of mucus and pulmonary surfactant, which act as physical barriers to lung delivery (discussed further in later sections) and can vary greatly across patients and pathologies ${ }^{118,119}$. Furthermore, a recent comparison of three widely used routes of pulmonary administration in mouse models - intratracheal instillation, intratracheal spraying and intranasal instillation - revealed different rates of polymeric (PLGA) NP deposition in the lungs and heterogeneous distributions overall, suggesting the need for validated and consistent delivery methods when assessing pulmonary administration routes for $\mathrm{NPs}^{120}$. Clinically, approved NP formulations (such as ONPATTRO, VYXEOS and NBTXR3) are either intratumourally or intravenously administered, with limited optimization of the administration route ${ }^{7}$. Although preclinical work is being done to explore alternate routes, these studies are still ongoing. In all, selecting the optimal administration route for NPs may allow for more desirable distribution, but many current administration routes still, ultimately, result in widespread distribution of NPs and fail to provide the level of targeting and specificity desired.

To further prevent non-specific distribution, many NP platforms have added targeting moieties to their surface to direct their delivery. Most targeting moieties including antibodies ${ }^{121}$, glucose $\mathrm{e}^{122}$, transferrin $^{34}$, folate ${ }^{123}$, transporters ${ }^{2}$ and integrin ligands ${ }^{124}$ - use interactions with molecules on the target cell's surface, such as ligand-receptor, enzyme-substrate or antibodyantigen-mediated interactions ${ }^{73}$. Thus, targeted NPs must be engineered with a targeting moiety density that allows for these cell surface interactions, making it important to understand the ratio of receptors to ligands and the number of interactions needed to overcome the initial energy barrier to NP uptake $e^{2,76}$. Active targeting may also improve NP distribution within a target tissue: binding peptides for collagen type III increased $\mathrm{NP}$ accumulation in joints and enabled preferential NP association with osteoarthritic cartilage over healthy tissue $^{26}$. Additionally, use of the tumour-targeting peptide CREKA allowed for enhanced permeation and uniform distribution of NPs in a mouse model of breast cancer with solid tumours ${ }^{125}$. However, despite the benefits of active targeting, the process of target selection is limiting. Because disease markers can vary among diseased cells within a patient as well as among patients in a population, target selection is a personalized process ${ }^{73,76}$. Furthermore, although antibodies can be engineered with high specificity, their conjugation to NPs may increase MPS interactions and result in rapid NP clearance $^{76}$. Although the selection of less specific targets, such as broadly expressed transporters, may reduce immunogenicity compared with antibodies, they face additional obstacles associated with off-target delivery ${ }^{2}$, which occurs if the marker or target is expressed on both diseased and healthy cells. Off-target delivery is further complicated if the diseased cells are widely distributed throughout normal tissues, which precludes local delivery ${ }^{61,73,97,126}$. Overall, although targeting NPs to disease markers aids in specific delivery, active targeting is not currently an ideal solution.

Physical barriers to NP distribution include tight junctions among the endothelial and epithelial cells of the BBB (in intravenous delivery) and the gastrointestinal tract (in oral delivery), respectively. For NPs to reach the central nervous system (CNS), they must utilize receptor-mediated endocytosis to be taken up by endothelial cells of the BBB and exocytosed to the

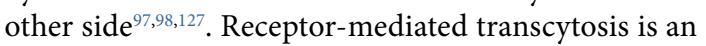
effective way to deliver therapeutics to the brain or to infiltrate tumour tissue ${ }^{128,129}$. However, this method of crossing the $\mathrm{BBB}$ is complicated by the heterogeneity of plasma membrane transporters on endothelial cells ${ }^{2}$. However, some transporters - such as glucose transporters - are consistently highly expressed on the $\mathrm{BBB}$, and some common targets - such as vascular cell adhesion molecule 1 - can increase NP transport across the $\mathrm{BBB}^{2,91}$. These two molecules could be harnessed to deliver NPs. Other targeting routes have been explored, including the transferrin receptor, which has theoretical advantages over other transporter types but has yet to see clinical success ${ }^{130}$. With transferrin receptor systems, only approximately $5 \%$ of the systemically administered NP dose reaches the CNS and even less reaches target cells ${ }^{97,127}$. However, a recent investigation characterizing AuNPs that had crossed the BBB revealed that the composition of the NP corona was altered but stable after crossing: investigations to better understand these altered coronas could help develop future strategies for CNS targeting ${ }^{96}$. Overall, the BBB remains a major challenge for systemically administered NPs attempting to reach tissues of the CNS. Thus, intranasal administration has been increasingly explored as an option for NP delivery to the brain as it bypasses the BBB and avoids many of the limitations of systemic delivery ${ }^{115,131}$. However, factors such as a limited dosing volume and variables attributed to patient congestion and mucus have presented notable obstacles to the intranasal route ${ }^{132,133}$.

Although oral delivery is the most widely used and readily accepted form of drug administration, the gastrointestinal tract presents numerous barriers for $\mathrm{NPs}^{72}$. For NPs that rely on passive diffusion, crossing the endothelium is restricted by concentration gradients and P-glycoproteins that excrete drugs from the vasculature into the intestinal lumen. However, some NP properties may encourage transport across the gastrointestinal tract. In a recent screen of inorganic NPs for the oral delivery of protein drugs, smaller, negatively charged silica NPs enhanced intestinal permeation by opening tight junctions, thus avoiding the need for cellular uptake for 
Stenosis

The narrowing of a bodily passage (such as a blood vessel). transport across the epithelial barrier ${ }^{134}$. However, for platforms that rely on endocytosis and subsequent exocytosis to cross the gastrointestinal tract, size remains an important factor. For example, the large surface area of polymeric NPs (as compared with soluble drug) has been beneficial as it increases the number of interactions with the gastrointestinal tract following oral delivery ${ }^{135}$. Overall, the average optimal reported size for NP transcytosis in gastrointestinal applications seems to be around $100 \mathrm{~nm}^{28,48,135-137}$. This size range allows for both enterocytes and $\mathrm{M}$ cells - which preferentially take up NPs $20-100 \mathrm{~nm}$ and $100-500 \mathrm{~nm}$ in diameter, respectively - to transport NPs across the gastrointestinal $\operatorname{tract}^{47}$. Rod-shaped NPs generally outperform spherical particles, which aligns with trends showing that nanorods are internalized into epithelial cells more efficiently than spheres are ${ }^{135,138,139}$. However, even when NPs are internalized by intestinal epithelial cells, only a small percentage undergo exocytosis ${ }^{140}$. Thus, even when utilizing these NP design elements to optimize transport, passive diffusion across the gastrointestinal tract is limited, so active targeting methods have been explored.

The transferrin pathway can be exploited for transepithelial movement in the intestine, using a transferrincoated $\mathrm{NP}^{136}$. This target may be especially useful in the treatment of colon cancer and irritable bowel disease, which both cause overexpression of the transferrin receptor in the intestinal mucosa ${ }^{136}$. However, in addition to the limitations of active targeting described above, targeting strategies in the gastrointestinal tract are frustrated by the formation of coronas in gastrointestinal fluids, which vary with diet, and goblet cells that produce mucus to coat the endothelial surface. Both of these issues limit the interactions between NPs and the intestinal walls ${ }^{72,141}$. These barriers are made heterogeneous by pathologies, such as inflammatory diseases, that may increase epithelial permeability and alter mucus production, $\mathrm{pH}$ and the gastrointestinal microbiome $\mathrm{e}^{72,142}$. Thus, the challenges presented by the gastrointestinal tract, and heterogenized by patient pathologies, present substantial barriers to achieving therapeutically desired biodistribution via oral delivery.

\section{Microenvironmental barriers}

Once at the target site, NPs must navigate the local microenvironment. Here, obstacles may include changes in chemical conditions or physical barriers to penetration. Thus, to successfully engineer NPs that reach the desired tissues or cells, a fundamental understanding of the microenvironments they will encounter is critical

Microenvironment variability. Microenvironments often feature conditions that are substantially different from those in the circulation, which can greatly alter the physical properties and stability of NPs. For example, the gastrointestinal tract includes areas of extreme $\mathrm{pH}$ variation and acidity ${ }^{72}$. These conditions, in addition to the presence of enzymes that induce degradation, make the gastrointestinal tract an unstable environment for many NPs ${ }^{72,74}$. Furthermore, the gastrointestinal microenvironments can be diversely altered by disease states, resulting in heterogeneous reactions to biomaterials $^{74}$. For example, a comparison of microenvironments in colon cancer and colitis, which feature different amine surface group densities on colon tissue, determined that the pathologies resulted in diseasedependent compatibility with dendrimer/dextran biomaterials ${ }^{74}$.

Numerous diseased microenvironments feature variations in $\mathrm{pH}$, such as the low $\mathrm{pH}$ observed in many tumours or the fluctuating $\mathrm{pH}$ observed across stages of wound healing ${ }^{90,143}$. Some $\mathrm{pH}$-sensitive NP platforms (detailed below) have been developed that allow the release of the drug only in specific $\mathrm{pH}$ conditions. Wound sites are often hyperthermic, so temperature-responsive systems can react to this local environment and provide targeted delivery ${ }^{144}$. In the case of stenosis and atherosclerosis, narrowed arteries result in elevated shear stresses that can be exploited to increase therapeutic release from NPs that break down under these conditions ${ }^{145}$.

Local NP distribution. Barriers to local distribution have been explored in depth in the tumour microenvironment, as NP penetration and stability are challenging in solid tumours ${ }^{107,146}$. Many characteristics of the tumour microenvironment - including the vasculature, interstitial fluid pressure and extracellular matrix (ECM) density - contribute to the limited permeation and penetration of $\mathrm{NPs}^{3,147-150}$. Thus, the exact cause of successful NP accumulation in tumours has been highly debated, with only a few established trends correlating NP design to tumour delivery. Some of these NP properties that can promote accumulation in tumours (FIG. 3) include hydrodynamic diameters above $100 \mathrm{~nm}$, rod-shaped architectures, near-neutral charges or inorganic material compositions - all of which may be optimal for tumour accumulation $^{71}$.

The tumour microenvironment also plays a key role in determining NP fate. As the vasculature within tumours is heterogeneous and abnormal, NPs can accumulate in tumours as the leaky vessels enable NP extravasation, a phenomenon often referred to as the enhanced permeation and retention (EPR) effect ${ }^{61}$. Reports vary on the role of the EPR effect in NP accumulation in tumours. Up to $10-15 \%$ of injected NPs accumulate at the tumour site, as compared with $0.1 \%$ of free drug, and some studies attribute this to the EPR effect $^{61}$. In contrast, recent work utilizing a combination of computational analysis and imaging techniques in a mouse tumour model has determined that only a fraction of NP accumulation in tumours can be attributed to passive transport, including the EPR effect. Instead, the work suggests that other mechanisms such as immune cell interactions, protein coronas and molecular mechanisms may contribute substantially to the enhanced tumour accumulation of $\mathrm{NPs}^{129}$. These conclusions seem to be supported by meta-analysis: one study reviewed 232 data sets and determined that, on average, only $0.7 \%$ of injected NP doses reach tumours - a finding that greatly de-emphasized the impact of the EPR effect ${ }^{71}$. However, it is important to note the limitations of these generalized findings, as a recent investigation has highlighted the potentially misleading results from quantifying NP distribution using non-standard calculations, 
which may have led to biased results ${ }^{151}$. Thus, while continuing to explore the broad implications of the EPR effect for NP accumulation, future investigations must critically evaluate the metrics used to quantify delivery and distribution.

The EPR effect relies on the heterogeneous formation of vasculature throughout tumours that can be altered by individual patient factors such as age, genetics, lifestyle and even previous antitumour treatments ${ }^{61,98,146}$. Thus, to select the appropriate delivery platform for a specific patient, their individual tumour and its vasculature should be assessed for EPR effects that alter NP accumulation and permeation ${ }^{61,106}$. This is a promising diagnostic application for tagged NPs, which have been used in preliminary studies to quantify the level of the EPR effect at the tumour site seen in individual patients in an attempt to identify patient populations that are well suited for NP-based therapies ${ }^{152}$.

The heterogeneity of tumour microenvironments generates many obstacles to successful NP delivery, including reduced permeation. Within the tumour environment, cells may overproduce or generate altered ECM components that result in a dense ECM that physically hinders NP delivery ${ }^{147-149}$. This is especially true for cationic NPs as they adhere to the negatively charged tumour ECM, reducing permeation ${ }^{71,153}$. In addition, abnormalities in tumour lymphatic vasculature can result in decreased interstitial fluid drainage, which increases intertumoural interstitial pressure and prevents effective NP perfusion ${ }^{3,107,148,150}$. These barriers can prevent most tumour cells from interacting with NPs; one study found that antibody-targeted NPs interacted with only $2 \%$ of tumour cells - a number far below the level required for therapeutic efficacy ${ }^{148}$.

Limited perfusion is a therapeutic obstacle for NPs delivered to the brain as well. After crossing the BBB via systemic delivery or local administration, NPs in the brain microenvironment often fail to permeate the tissue because of the limited extracellular space and non-specific adherence to the $\mathrm{ECM}^{154,155}$. Thus, advanced delivery methods such as convection-enhanced delivery, and NP surface modifications, such as dense PEG coatings, have been explored. These methods may aid in more widespread and evenly distributed delivery across the brain, as well as improved permeation in glioblastomas ${ }^{154-156}$.

NPs face additional barriers to local distribution, including biofilms and mucus ${ }^{72,149}$. Within mucus layers, the distances between adjacent polymer links determine the mesh pore size, which can vary from 10 to $1000 \mathrm{~nm}$, so smaller objects diffuse through whereas larger objects are trapped ${ }^{72,149}$. In addition to filtering by size, mucus may trap objects via non-specific interactions that lead to their rapid clearance from epithelial surfaces $^{72}$. Although mucus throughout the body shares a similar function, its behaviour varies depending on its physiological location because of differences in its composition, hydration and viscoelasticity ${ }^{72,119,157}$. For example, mucus in the gastrointestinal tract acts as an adherent, thick layer whereas mucus in the lungs tends to be thinner and more mobile, making it a heterogeneous barrier $^{72,119,157}$ (FIG. 3).
Although mucus behaviour can be generalized within each of these physiological environments, there are areas of disparity within the mucus of an organ system, and these barriers are dynamic. In the gastrointestinal tract, the thickness of the mucus barrier can range from 40 to $450 \mu \mathrm{m}$ in the stomach and from 110 to $160 \mu \mathrm{m}$ in the colon, and factors such as fibre intake affect both mucus

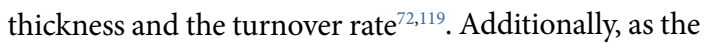
mucosal barrier transitions between the near-neutral endothelial cell surfaces and the acidic intestinal lumen, a steep $\mathrm{pH}$ gradient is present across its micrometre-scale thickness, creating a very unstable environment for NP platforms $\mathrm{s}^{72,74}$. Changes to these properties of the mucus in the gastrointestinal tract are also observed in pathologies that change glycosylation patterns, $\mathrm{pH}$ and the mucus layer thickness ${ }^{72,142}$.

Similarly, pathologies of the lungs change mucus behaviour in that tissue. Mucus in the lungs - a barrier that greatly impacts inhaled NPs - is characterized by high concentrations of MUC5AC and MUC5B polymers $^{118,157}$. However, in cystic fibrosis, increased MUC5B expression and excessive cross-linking of polymers in the mucus results in decreased pore size and low rates of mucus clearance because this mucus has higher viscosity, which encourages biofilm formation by entrapping pathogens and limiting the mobility of neutrophils ${ }^{157,158}$. MUC5B concentrations are also elevated in cases of primary ciliary dyskinesia and cigarette smoke-induced chronic bronchitis; MUC5AC is elevated in asthma ${ }^{158}$. In all, the properties of mucus have been found to vary greatly based on patient factors such as diet, lifestyle and disease, making it a complex environment for inhaled NP delivery.

\section{Cellular and intracellular barriers}

When NPs make contact with their target cells, there are still numerous barriers to the uptake and intracellular trafficking that determine their functional delivery ${ }^{3}$. This section explores the barriers NPs must overcome to achieve cellular uptake and proper internal trafficking and discuss how cellular heterogeneity affects these NP interactions.

NP uptake and internalization. The corona, in combination with the NP characteristics it alters, such as hydrophilicity and charge, alters cellular uptake in numerous cell types including macrophages and cancer cells $s^{61,159,160}$. This corona-covered NP interacts with the surface of the cell, which consists of a negatively charged, selectively permeable phospholipid bilayer with biomolecules incorporated throughout in a fluid mosaic structure ${ }^{75,160}$. Cell membranes vary widely and membrane components such as lipid rafts and transmembrane proteins are heterogeneously distributed; over 400 cell surface transporter types have been identified in human cells $s^{2,75,160}$. Furthermore, the exact stiffness of the cell membrane and its compositional fluidity are determined, in part, by the cytoskeleton, which can respond to external cues, making these characteristics dynamic ${ }^{161}$. Thus, NPs interacting with the same cell may experience different interactions depending on their location on the cell's membrane or their time of contact. Anionic NPs may struggle to make contact 
with the cell surface due to repulsive forces, whereas cationic NPs, if too positively charged, may damage the cell membrane and even cause cytotoxicity ${ }^{3,76,159,162}$. Thus, the first contact between a NP and a cell - which varies with NP and cell properties - may determine the NP fate and, therefore, its therapeutic potential.

For the next step in delivery - cell uptake - few definitive trends have been established concerning the optimal NP shape and size; some models and studies indicate that, in non-phagocytic cells, spherical NPs have improved uptake over rod-shaped particles ${ }^{163,164}$, but other studies show the opposite effect ${ }^{165,166}$. Similarly, many in vitro studies have shown that non-phagocytic cells only take up NPs that are $10-60 \mathrm{~nm}$ in size, and that smaller NPs internalize better, whereas other investigations indicate that smaller NPs are more likely to cause cytotoxicity $^{2,76,167}$. The process of NP uptake can be broken down into passive and active methods ${ }^{75}$. Because the cell membrane is selectively permeable, passive diffusion is predominantly limited to small, uncharged molecules that travel down concentration gradients ${ }^{162}$. Thus, NPs most commonly rely on active transport to cross the cell membrane ${ }^{3,75}$. Specifically, NPs tend to utilize endocytic pathways, in which the plasma membrane is folded into vesicles to engulf NPs on the cell surface, and then release them intracellularly ${ }^{3,75,90,160}$. The type of endocytosis a NP undergoes can affect its fate within the cell and is determined by numerous factors including cell type, NP size and receptor interactions $s^{3,90,160}$. For example, in non-specific cell membrane interactions, smaller or larger NPs will be taken up by either phagocytosis or pinocytosis, respectively ${ }^{90}$.

However, more specific interactions - often with negatively charged NPs - may result in caveolin-mediated or clathrin-mediated endocytosis ${ }^{162}$. Caveolin-mediated endocytosis can occur in molecules smaller than approximately $60 \mathrm{~nm}$ and utilizes lipid rafts to create specialized vesicles after engulfment ${ }^{90}$. This form of endocytosis is more common for nanorods; nanosphere uptake is usually clathrin-mediated ${ }^{138}$. Clathrin-mediated endocytosis the most common route for NP uptake in non-specialized mammalian cells - relies on receptor-mediated, hydrophobic or electrostatic interactions between NPs and the cell membrane in areas of clathrin expression ${ }^{90,160}$. The induction of these endocytic pathways is influenced by NP properties such as stiffness and size. Although results vary, stiffer NPs are generally more easily taken up, and both experimental and theoretical analyses indicate that endocytosis of rigid particles requires less energy ${ }^{162,168}$. Additionally, NPs that are too small $(<30 \mathrm{~nm})$ may not be capable of driving membrane wrapping enough to activate endocytic processes ${ }^{76}$. Multiple studies report good cellular uptake and intracellular delivery when particles $\sim 50 \mathrm{~nm}$ in diameter are used ${ }^{27,76,169-172}$. Thus, the process used for NP uptake is determined by numerous factors including characteristics of the cell membrane as well as properties of the NP, which also influence the subsequent endocytic process (FIG. 4).

During endocytic processes, the vesicles, or endosomes, go through different stages that involve changes in their chemical composition and $\mathrm{pH}$ until they become lysosomes, which feature low $\mathrm{pH}$, high ionic strength and proteolytic enzymes that affect the stability of NPs and their cargo ${ }^{75,160}$. Materials that change in response to acidic conditions and have a proton sponge effect have been investigated to aid in endosomal escape, enabling NPs to avoid degradation ${ }^{40,169,173}$. LNPs, which include cationic and ionizable materials, are good examples of these intracellularly triggered delivery mechanisms and are often used to carry nucleic acids into cells ${ }^{3,174-179}$. Materials can respond to the acidic endosomal $\mathrm{pH}$, but NPs have also been designed to react to the reductive endosomal environment ${ }^{180,181}$. As the redox potential of the endosome increases, cleavable linkers incorporated into the NP design may allow the NP to degrade, disrupt the endosomal membrane and release its cargo intracellularly ${ }^{180,181}$. In addition to responsive NPs, complex shapes, such as nanostars, have also been shown to improve the intracellular delivery of genetic material as they can efficiently enter cells and escape endosomes ${ }^{172}$.

Once in the cytosol, the cargo may still need to reach certain intracellular environments ${ }^{75,160,161}$. Because cells are highly compartmentalized, reaching these organelles may require crossing additional intracellular membranes ${ }^{161}$. For example, the nuclear membrane is a barrier for genome editing or DNA delivery ${ }^{75,149}$. NPs targeting the mitochondria for specific cancers or as neurogenerative or cardiovascular therapies face similar barriers ${ }^{75,182}$; to overcome this challenge, $\mathrm{pH}$-responsive NP systems could aid in precise delivery to the mitochondrial environment ${ }^{183}$.

Cellular heterogeneity. In addition to the general cellular barriers described above, cells form heterogeneous populations both within a patient and across a patient population. Many cellular variations occur based on the characteristics of an individual. For example, in human fibroblast cells from fetal lungs and epithelial cells from fetal colons, younger cells took up more NPs than old cells, and younger cells were less susceptible to toxicity ${ }^{184}$. Additionally, a study found that cell sex altered the uptake of AuNPs in human amniotic stem cells and fibroblasts isolated from saliva, demonstrating yet another factor to consider in NP delivery ${ }^{185}$.

Drug-resistant cells contribute to the cellular heterogeneity that challenges NP delivery ${ }^{186}$. For example, resistance to platinum (II)-based drugs, such as oxaliplatin and cisplatin, which distort DNA structure to induce apoptosis, can occur if cancer cells overexpress efflux pumps or increase their rate of DNA repair. Thus, smart NP platforms must be engineered to overcome these barriers. For example, micelles deliver NPs more effectively to the nucleus, and thus the cell has fewer opportunities to acquire drug resistance ${ }^{187,188}$. Thus, both cell type and acquired phenotypes that lead to a heterogeneous cell population create diverse barriers to NP delivery, but new developments in NP design may help overcome these obstacles.

\section{NPs in precision medicine}

To account for the vast heterogeneity of biological barriers and disease states within and across patient populations, methods must be developed to deliver therapeutics in a manner that is highly modular and customizable. 

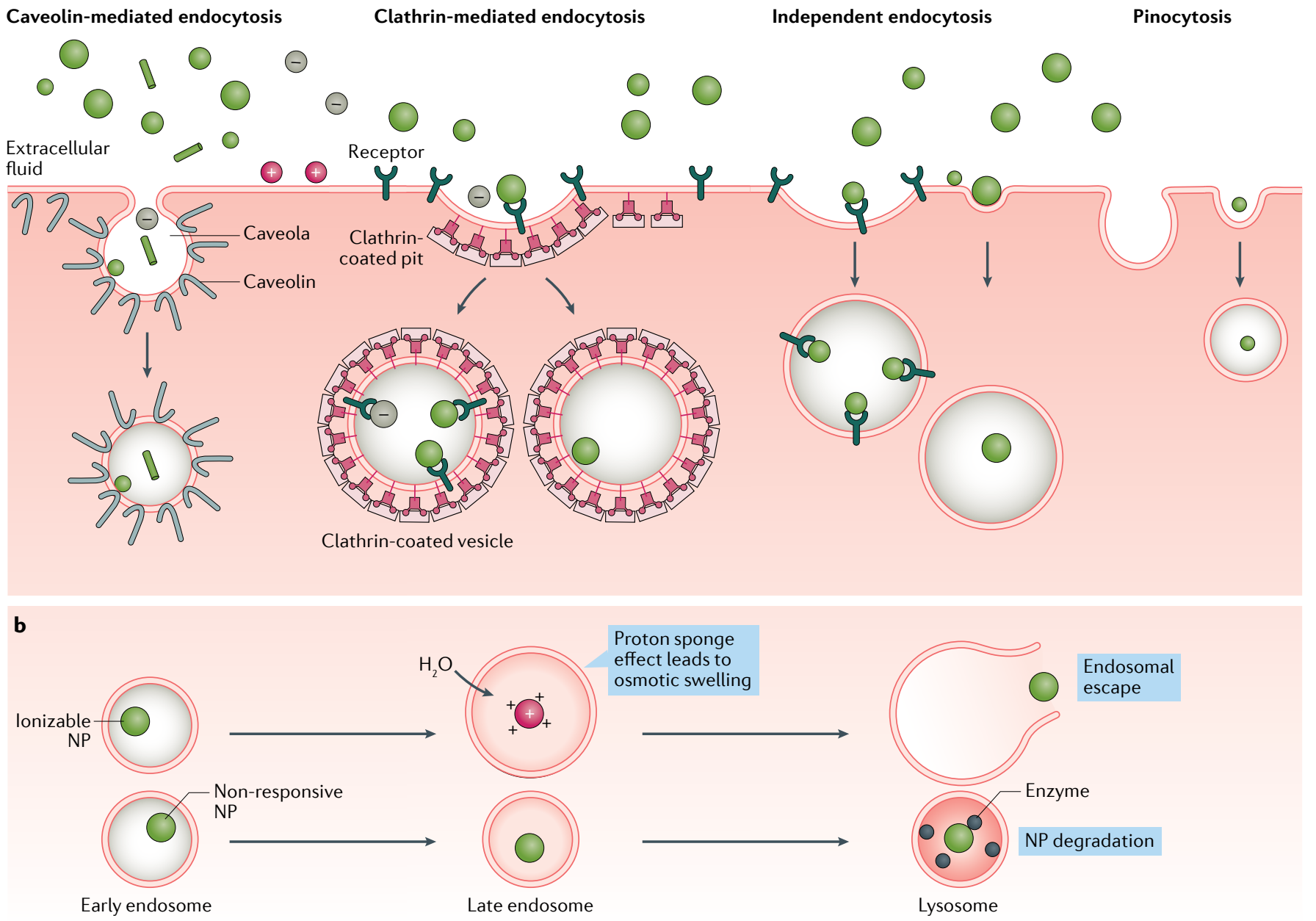

Fig. 4 | Common uptake pathways that ultimately determine NP fate within a cell. a | Upon interaction with the cell surface, nanoparticles (NPs) - depending on their surface, size, shape and charge - are taken up by various types of endocytosis or pinocytosis via non-specific interactions, such as membrane wrapping, or specific interactions, such as with cell surface receptors. $\mathbf{b}$ | Once they have entered the cell, NPs remain trapped within vesicular compartments, or endosomes, that feature various characteristics such as internal or external receptors. To achieve functional delivery, most NPs must escape from these compartments before they acidify. Thus, responsive NPs — such as ionizable NPs that become charged in low-pH environments - aid in endosomal escape and allow for intracellular delivery whereas unresponsive NPs often remain trapped and are destroyed by lysosome acidity and proteolytic enzymes.
This section details the effects of various NP properties on delivery, with a focus on how individual NP design choices (such as architecture, material properties, targeting and responsiveness; FIG. 5) can overcome barriers specific to individual diseases and patients.

\section{NPs for cancer therapy}

Cancer remains the second leading cause of death worldwide $^{189}$. Cancer is heterogeneous, and the development of effective cancer therapies is very challenging partially because of this complexity. However, precision medicine has emerged as a promising approach, and targeted chemotherapeutics have been developed that can treat patients who express specific biomarkers. The first drug of this type, imatinib (Gleevec; Novartis), is given to patients with chronic myeloid leukaemia who express the $\mathrm{BCR}-\mathrm{ABL}$ fusion protein from the Philadelphia chromosome ${ }^{190}$. FDA approval of imatinib opened the field for many other successful targeted chemotherapeutics ${ }^{190-192}$. However, these therapies and others could be more effective if delivery is improved. For example, imatinib has also been delivered using a NP system, which enhanced tumour accumulation and regression in vivo, improving the survival ratio to $40 \%$ after 60 days in a melanoma mouse model ${ }^{193}$. Improvements in delivery could overcome some limitations of therapeutics that have failed to make it to the clinic, including small-molecule drugs with limited water solubility or antibodies with low stability ${ }^{194}$. Similarly, many chemotherapeutics have off-target toxicity and induce adaptive resistance, which limit efficacy. Furthermore, there are many biological barriers associated with cancer, specifically at the tumour site. Improved delivery techniques could offset many of these concerns. In order to best leverage our knowledge and treatment of individual cancer patients, both therapeutics and their delivery systems can be personalized for a given patient. 
Adapting to the tumour microenvironment. The tumour microenvironment heavily influences patient prognosis, as it affects chemotherapeutic efficacy ${ }^{195}$. Although the EPR effect and FDA approval of early NP systems has given hope for NP-based delivery, these early systems do not improve overall patient survival, and there is still significant work to be done using smart NP designs to improve cargo delivery or remodel microenvironments and thus increase the efficacy of existing therapies ${ }^{69}$.

For example, incorporating cell membranes into NPs can improve their accumulation in cancerous tissue. NPs wrapped with membranes that are harvested from a patient's own cancer cells homotypically adhere to patient-derived cancer cell lines; mismatch between the donor and host results in weak targeting ${ }^{196,197}$. NPs wrapped with macrophage or leukocyte membranes recognize tumours, and hybrid membranes, such as erythrocyte-cancer cell hybrids, can further increase specificity ${ }^{197-199}$. NPs that utilize these membranes show a twofold to threefold increase in drug activity over the free $\operatorname{drug}^{198}$. In a similar fashion, material properties can cause NPs to preferentially distribute to certain tissues. For example, a poly ( $\beta$-amino-ester) (PBAE) ter-polymer/PEG lipid conjugate was optimized for lung localization, achieving efficacy two orders of magnitude above the pre-optimized form both in vitro and in vivo ${ }^{179}$. Other PBAE polymers have been developed that preferentially target glioblastoma cells over healthy cells in vitro ${ }^{200}$. Even AuNPs can be optimized to passively target triple-negative breast cancer cells,

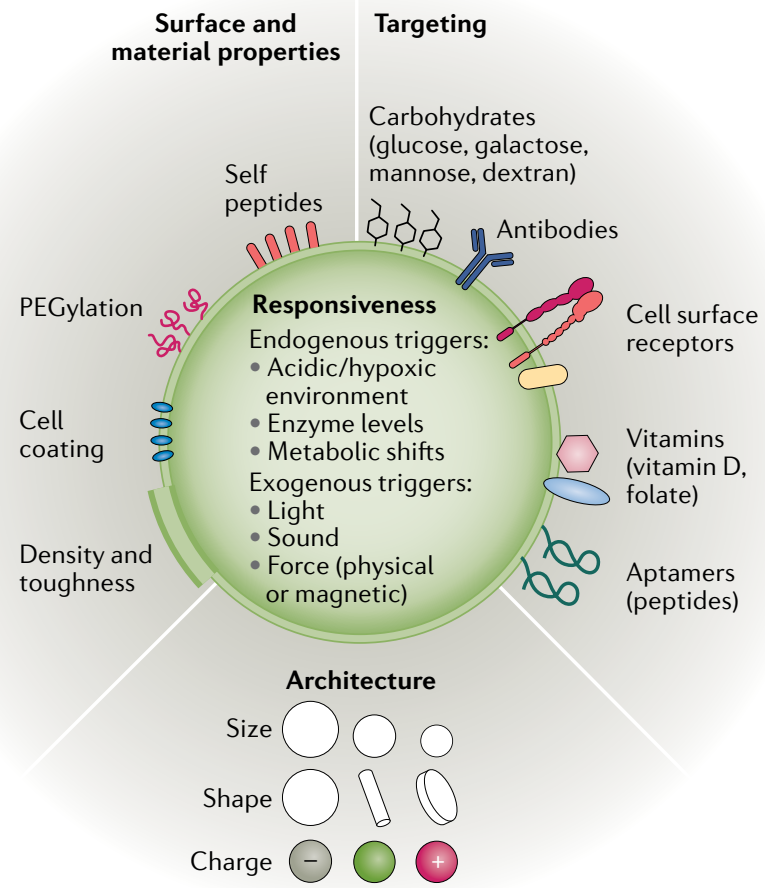

Fig. 5 | Commonly engineered NP surface properties that allow for enhanced delivery. Surface and material properties, architecture, targeting moieties and responsiveness are all attributes of nanoparticles (NPs) that can be altered in intelligent designs to tailor the platform to a specific application. Different combinations of these four properties allows for seemingly endless permutations of NP features and platforms. PEG, poly(ethylene glycol). which notoriously lack traditional cell surface targets ${ }^{201}$. Designs like these, as well as the more generalizable trends for NP size and shape, are being used to improve the percentage of chemotherapeutic dose that makes it to the solid tumour site.

Within the tumour microenvironment, responsive particles can improve tumour penetration, overcoming the high interstitial pressure and dense ECM that typically prevent NP permeation ${ }^{150,202}$. Endogenous triggers - such as the acidic and hypoxic environment of the tumour - can be used to induce NP degradation and drug release $\mathrm{e}^{147,150,203,204}$. High enzyme levels of matrix metalloproteinases (MMPs) and other extracellular proteases can serve as triggers ${ }^{10,205-207}$, and the Warburg effect, a metabolic shift towards anaerobic glycolysis ${ }^{195}$, can be exploited as well ${ }^{208}$. Exogenous triggers - such as light, sound waves, radio frequencies and magnetic fields - can also be used and tightly controlled from outside the body ${ }^{206}$. A non-invasive existing clinical technique, ultrasound, can trigger local release from a systemically administered particle ${ }^{68,209}$. Near-infrared light, another exogenous trigger, has low absorption by natural tissues and therefore good biocompatibility ${ }^{210,211}$. Regardless of the trigger type, chemotherapeutics delivered locally in this responsive fashion have fewer off-target toxicities and other negative systemic effects.

One example of smart NP design, iCluster, is a stimuli-responsive clustered NP system that breaks down into smaller and smaller pieces as it overcomes biological barriers in the tumour environment ${ }^{204}$. The initial size of $\sim 100 \mathrm{~nm}$ favours extended circulation in the bloodstream and capitalizes on the EPR effect as the NP extravasates through the tumour vasculature ${ }^{204}$. At the tumour site, the low $\mathrm{pH}$ triggers breakdown of the system into much smaller ( $\sim 5 \mathrm{~nm})$ dendrimers, which have improved tissue penetration and thus deliver more of the platinum chemotherapeutic cisplatin to cancer cells $^{204}$. This system is a vast improvement over the traditional intravenous administration of free cisplatin: administration of the free drug inhibits tumour growth by $10 \%$, whereas the iCluster system inhibits growth by up to $95 \%$ in in vivo studies ${ }^{204}$. Additionally, free cisplatin commonly causes irritation and cytotoxicity, especially in the kidney. Size-switching is not a unique property of this system, and has been achieved using various other triggers and materials $\mathrm{s}^{10,207,212,213}$. NP systems such as these have great potential to improve therapeutic efficacy; their design is versatile and can be tailored specifically to the tumour microenvironment.

Another example of optimally designed delivery is a poly(acrylamide-co-methacrylic acid) nanogel, which can be modified with bioactive moieties for numerous applications including local $\mathrm{pH}$ response, cell targeting, transduction of visible light for photothermal therapy or degradation in the intracellular environment ${ }^{11}$. This platform was able to maintain the function of multiple modifications, allowing for each added small molecule, peptide or protein to contribute new responsive or recognitive properties ${ }^{11}$. Nanomaterials that utilize a similar, modular approach could be rapidly designed to deliver multiple therapeutic agents intracellularly or respond to sequential biological stimuli. 
Active targeting to cancer cells. Existing chemotherapeutics have various mechanisms and sites of action. Some disrupt DNA within the nucleus (doxorubicin, platinum drugs), and others work within the cytosol or affect organelles such as the mitochondria ${ }^{214}$. Each drug must be delivered to its site of action at therapeutic levels in order to work properly, indicating a need for NP trafficking to these sites.

Antibodies, carbohydrates and other ligands on the NP surface can induce specific and efficient NP uptake $^{124}$. Examples of tumour cell targeting moieties include antibodies ${ }^{121}$, peptides ${ }^{126}$, integrin ligands ${ }^{124}$, glucose $^{122}$, transferrin ${ }^{34,215}$ and folic acid ${ }^{123}$ (FIG. 5). As these technologies advance, some systems now incorporate multiple targeting modalities in a single $\mathrm{NP}^{195}$. Whereas some of these targeting schemes are generalizable, such as folic acid (folate receptors are overexpressed on $>40 \%$ of human cancers $)^{216}$, most require tumour profiling to establish receptor or ligand overexpression. Additionally, not all receptor targeting improves specificity. Some receptors overexpressed in tumour cell lines are also expressed in healthy tissues, limiting efficacy.

There is also often a trade-off between residence time in the circulation and cellular uptake. Recently, NPs have been developed with detachable stealth corona systems and charge-reversal systems (negative or neutral charge for circulation, positive charge for uptake), in an attempt to optimize both properties ${ }^{217,218}$. One such system utilizes an MMP-degradable linker to attach PEG to the surface of the NP: in the tumour microenvironment, the PEG coating is degraded, exposing a cell-penetrating peptide $^{219}$. In this way, systems can be developed that change a given property to optimize for the delivery barrier they currently face.

\section{NPs for immunotherapy}

Although immune checkpoint inhibitors have shown significant promise for cancer treatment ${ }^{220}$, there are still challenges with efficacy, patient variability and off-target effects when immunomodulators are used ${ }^{221}$. Some immunotherapeutics, such as proteins, have limited delivery potential when administered freely, and thus NPs have the potential to significantly improve delivery by protecting immunotherapeutics and enhancing their interaction with immune cells ${ }^{222}$.

Immune activation. The immune system is trained to eliminate cancerous cells from the body, but certain genetic traits can allow cancerous cells to evade and suppress immune cells. To resensitize these cells, cancer vaccines aim to train the body to recognize cancerous cells by using antigens either from the patient or from allogenic tumour cells. For example, Sipuleucel-T, an FDA-approved cancer vaccine (albeit with limited effi(acy) $)^{221}$, utilizes recombinant antigens specific to the tumour type. Although the drugs are not yet in the clinic, other groups have also developed synthetic peptides and tumour lysates with the ultimate goal of patient personalization $^{223-225}$. NPs can protect these antigens from degradation, improve the likelihood that they are presented to target immune cells and reduce off-target effects. Antigen-presenting cells (APCs) that take up these NP systems present the antigen cargo to T cells to prime and activate them. NPs used in these systems can be polymeric (PLGA) ${ }^{226}$, lipid-based (liposomes, LNPs) $)^{227,228}$, inorganic (gold, silica) $)^{229,230}$ or biologically derived (cell-membrane vesicles) ${ }^{231,232}$. NP-based cancer vaccines are currently being used in clinical trials ${ }^{233}$. Recently, NPs have been extensively explored in vaccines against SARS-CoV-2 (which causes COVID-19), with multiple successful late-stage clinical trials. Companies such as Moderna and BioNTech use LNPs to encapsulate mRNA that encodes for a COVID-19 antigen. As of 30 November 2020, Moderna and BioNTech/Pfizer have met their primary efficacy end points in phase III trials and have applied for Emergency Use Authorization. As with other applications, NP architecture, material properties and active targeting can affect cellular uptake, antigen presentation and the strength of the immune response $\mathrm{e}^{234}$.

Macrophages, B cells and dendritic cells are all APCs and can be targeted by NPs to improve the specificity of immune activation. Passive targeting includes optimizing size, shape ratios and using positively charged particles to interact with the negatively charged cell membranes ${ }^{235,236}$. APCs also express numerous carbohydrate-recognizing lectin receptors for endocytosis, and these have been exploited for cell-specific active targeting ${ }^{178}$. Some of these lectin receptors are expressed at high levels in certain APCs, such as the C-type lectin receptors lymphocyte antigen 75 (also known as DEC-205) and C-type lectin domain family 9 member A (CLEC9A), which can be used to target dendritic cells $^{237}$. Mannose is commonly used to target macrophages and tumour-associated macrophages ${ }^{238-240}$, but can target dendritic cells as well ${ }^{241}$. Particles coated with galactose, dextran or sialoadhesin can deliver to macrophages ${ }^{198,240,242}$. CD19-targeting NPs can be used to actively target $\mathrm{B}$ cells ${ }^{243}$, and NPs with lipoprotein surfaces can activate the scavenger receptor class B1 (SRB1) receptor on dendritic cells ${ }^{244}$. More generally, NP properties can be optimized for accumulation at tolerogenic organs, such as the liver and spleen, where immunological antigens are naturally produced ${ }^{245}$. Immune-recruiting systems, such as polymeric hydrogels and scaffolds, could also be used to optimize interactions with APCs. These systems work with APC-targeted NPs, allowing them to recruit and reprogramme APCs ${ }^{246}$. All of these methods aim to increase the likelihood that an antigen will interact with an APC, improving the efficacy of antigen-based therapies and lowering the dosage needed to reach therapeutic levels.

The stimulator of interferon genes (STING) pathway also leads to immune cell activation and antitumour effects, and can be activated by cytosolic double-stranded DNA (which typically comes from pathogens). STING agonists, typically cyclic dinucleotides, show promising antitumour activity, but are unstable and highly polar, which reduces cellular uptake ${ }^{247}$. NPs improve the delivery of STING agonists ${ }^{248-250}$; a single STING NP dose of one formulation increased survival for at least 80 days in mice ${ }^{249}$. Additionally, some NPs with cyclic structures (cyclic lipids) that mimic double-stranded DNA can stimulate STING regardless of their cargo ${ }^{174}$. 
Other immunotherapy approaches target $\mathrm{T}$ cells directly. Numerous targeting schemes have been used to target NPs to T cells. Examples include NPs targeting PD1 (REF. ${ }^{251}$ ), CD3 (REF. ${ }^{134}$ ) and THY1 (also known as CD90 $)^{135}$. The tLyp1 peptide, typically used for tumour targeting, has been used to target regulatory $\mathrm{T}$ cells, an immunosuppressive $\mathrm{T}$ cell subtype $\mathrm{e}^{193}$. Checkpoint inhibitors, an anticancer immune-boosting strategy, are typically monoclonal antibodies that target PD1, PDL1 or CTLA4. As for other applications, the usage of free antibodies is limited by stability concerns. Additionally, less than a third of patients who receive these checkpoint inhibitors see a robust response $\mathrm{e}^{249}$. In an attempt to improve these therapies by enhancing efficacy and reducing side effects, NPs have been formulated for monoclonal antibody (anti-PD1) delivery ${ }^{252,253}$, and other NP formulations disrupt immune checkpoints through the delivery of small interfering RNAs (siRNAs) ${ }^{254}$.

Genetically modified $\mathrm{T}$ cells have also shown promise in the treatment of metastatic and blood cancers. These $\mathrm{T}$ cells are constructed to express transgenic $\mathrm{T}$ cell receptors (TCRs) or CARs, which allow for T cells to specifically target and eliminate cancerous cells ${ }^{255}$. These T cells are extracted from patients before in vitro expansion using artificial APCs, and new NP formulations may allow for translation of this process in vivo ${ }^{255,256}$. Artificial APC design is similar to that of traditional NPs in the sense that their architecture, materials and targeting influence $\mathrm{T}$ cell activation ${ }^{257}$. Alternative methods of CAR T production could reduce the complexity of antigen delivery to T cells using NPs, including the delivery of CAR-encoding DNA in vivo and the delivery of CAR-encoding mRNA to produce transiently modified T cells ${ }^{258,259}$.

Immune suppression. Diseases such as rheumatoid arthritis and systemic lupus erythematosus also result from incorrect immune regulation: hyperactivation. In these autoimmune diseases, $\mathrm{T}$ cells and $\mathrm{B}$ cells are sensitized to self-antigens ${ }^{260}$. Autoimmune diseases are typically treated with general immunosuppressants, which can cause serious side effects. Conditions caused by immune overactivation could benefit from more targeted immunotherapies.

Cellular targets for immune suppression include $\mathrm{APCs}^{261}$, autoreactive $\mathrm{T}$ cells and $\mathrm{B}$ cells ${ }^{262}$, and regulatory $\mathrm{T}$ cells and $\mathrm{B}$ cells ${ }^{263,264}$. Antigen-specific immunotherapy aims to reprogramme or reduce reactive cells or impart them with tolerance to certain antigens $s^{260}$. By targeting a subset of immune cells, antigen-specific immunotherapy has potential to modulate the immune system without compromising systemic immunity. Passive and active targeting schemes similar to those used in immune-activating therapies are used for immune inhibition. For example, NPs coated with anti-CD2/ CD4 antibodies target T cells and can be used to increase the number of regulatory $\mathrm{T}$ cells in circulation, whereas non-coated NPs at equivalent doses could not ${ }^{263}$. Similarly, sialic acid-binding immunoglobulin-like lectins (Siglecs) can be used to target and induce tolerance in B cells ${ }^{262}$.

Immune tolerance can also be induced through the delivery of immunosuppressant agents. NPs that deliver
IL-2 and TGF $\beta$ can expand the number of regulatory $\mathrm{T}$ cells in vivo, suppressing the symptoms of lupus ${ }^{263}$. The active form of vitamin $\mathrm{D}_{3}$ has immunosuppressive effects because it modulates dendritic cell function ${ }^{261}$. Active vitamin $\mathrm{D}_{3}$ can cause hypercalcaemia when administered systemically, so NP delivery is a promising alternate strategy. PLGA NPs have been used extensively to deliver immunomodulators and prevent allograft rejection ${ }^{265}$; PLGA NPs anchored to a hydrogel allow for local and sustained (28-day) delivery of tacrolimus, a common immunosuppressant ${ }^{266}$. For more long-term effects, genetic engineering - reprogramming immune cells at the genomic level - could be effective ${ }^{267}$.

\section{NPs for genome editing}

Recent advances in CRISPR, transcription activator-like effector nuclease (TALEN) and zinc-finger nuclease (ZFN) technologies are making it increasingly easy to engineer the genome for widespread use in biomedical research, drug development and discovery, and gene therapy ${ }^{268}$. This is important in the context of precision medicine, as over 3,000 human genes have been associated with Mendelian diseases but less than 5\% of rare diseases have effective treatments ${ }^{268}$. Advances in genome editing are now making it possible to correct many of these rare diseases. However, efficient and safe delivery is still needed for genome-editing systems to effectively target and enter tissues and cells of interest, while also minimizing toxicity ${ }^{269}$. Delivery of genome-editing systems is challenging because these systems are multicomponent, hold sensitive cargo and need to overcome several extracellular and intracellular biological barriers to reach the genome of target cells. Lipid-based and polymer-based NPs have delivered a range of nucleic acids in vivo, and are in various stages of clinical development ${ }^{24,44,104,183}$. For example, a LNP siRNA drug termed Onpattro (patisiran) was recently approved by the FDA for the treatment of amyloidosis ${ }^{270}$. In the context of genome editing, NPs have the potential to be less toxic and immunogenic than viral vectors, which have a history of safety concerns s71,272. $^{27}$

Intracellular targeting. Most NP-based systems for genome editing are formulated by electrostatic complexation of nucleic acids with cationic materials, which are delivered intracellularly through mechanisms including receptor-mediated endocytosis and phagocytosis ${ }^{273}$. Cationic materials both complex with nucleic acids and impart responsive properties to NPs that aid in endosomal escape. Charged materials currently used for nucleic acid delivery include lipids (lipofectamine, rationally designed lipids, combinatorial libraries of ionizable lipid-like materials) $)^{21,22,274}$ and polymers (polyethylene imine (PEI), jetPEI, poly(amido amine) (PAA), polylysine (PLL), cyclodextrins and poly( $\beta$ amino esters) $)^{275-278}$. These systems are responsive to the intracellular environment, and can be optimized to incorporate passive and active targeting elements to ensure endocytic uptake.

The final destination of the cargo for RNA interference is the cytosol ${ }^{214}$. However, gene editing requires access to DNA. Strategies for nuclear targeting generally 
fall into two categories: using particles that are small enough to pass through the nuclear pore complex, or incorporating functionality that is used after endosomal escape ${ }^{214,279}$. NP properties can passively influence intracellular trafficking and the final destination ${ }^{179}$, but particles can also be actively targeted to specific intracellular sites and organelles, such as the mitochondria ${ }^{27}$. Defects within the mitochondrial DNA can also play a significant role in disease onset. However, success with mitochondrial DNA genetic engineering is currently limited to highly controlled in vitro settings ${ }^{214}$.

Applications of genome engineering. Cystic fibrosis is caused by genetic defects in the gene that encodes cystic fibrosis transmembrane conductance regulator (CFTR) protein. There is currently no cure for this life-threatening disorder, but it is a monogenic disorder and therefore amenable to gene therapy. In vitro, the CFTR gene can be replaced or precisely repaired ${ }^{280}$. However, gene therapy for cystic fibrosis has been largely unsuccessful in vivo due to issues with gene expression and delivery ${ }^{281,282}$. However, NPs could aid in overcoming these delivery barriers ${ }^{283-285}$. Multiple inhalable NP formulations have been developed, and some have shown successful delivery of genetic material such as mRNA $^{248,286}$.

Cystic fibrosis affects cells that produce mucus, making the mucus extra thick. This is the main symptom of the disease but is also a significant barrier to delivery. NPs have been developed with improved muco-penetrating properties for use in lung delivery for cystic fibrosis and for oral delivery. NPs smaller than the mucus mesh pores have improved penetration, as do systems with inert hydrophilic coatings (such as PEG or polyethylene oxide ${ }^{283,285}$. PEGylation has been shown to improve penetration through cystic fibrosis mucus ex vivo ${ }^{287}$. However, mucus can be highly variable between patients, and existing murine models may not accurately mimic the thickened airway mucus produced by patients with cystic fibrosis ${ }^{283}$. Other existing methods for improving mucosal delivery include the incorporation of muco-penetrating lipoplexes ${ }^{176}$, mucolytic proteins ${ }^{149}$, thiolated hyaluronic acid coatings ${ }^{48}$ and $N$-acetylcysteine ${ }^{137}$. All of these methods attempt to improve the transversal of mucosal barriers by altering NP surface properties ${ }^{118}$.

Even though it has been estimated that restoration of $10-35 \%$ of CFTR protein function would substantially improve the manifestations of the disease ${ }^{284}$, a higher percentage would be needed for a genuine cure. For genetic diseases such as these, the fetal stage is the most effective time for gene editing, as genetic defects are present in a small number of cells. However, fetal delivery is a challenge.

The biological barriers to in utero delivery are actually fewer than may be expected. NP therapies can be injected directly into an umbilical vessel, the amniotic fluid or specific fetal tissue ${ }^{288}$. The limitations in fetal delivery come from concerns regarding the interaction between fetus and mother. Viral vectors have successfully delivered DNA editing machinery in utero in a mouse model $^{289}$. However, these vectors have more toxicity concerns than NP systems do. Although the usage of NPs for this type of delivery is not widespread, there have been early successes with in utero NP delivery of peptide nucleic acids, resulting in a level of gene editing sufficient to alter the disease to manageable levels ${ }^{290}$.

Looking to the future, NPs have the potential to improve genome editing by exerting more precise control and reducing safety concerns. Several companies, including CRISPR Therapeutics, Intellia Therapeutics and Editas Medicine, are currently developing CRISPRCas9 therapeutics. Intellia Therapeutics is currently developing LNPs for in vivo delivery to treat several liver diseases, including amyloidosis, a1-antitrypsin deficiency and hepatitis B virus infection. With precision NP design, gene editing holds promise to cure diseases and significantly improve patient lives.

\section{Conclusions}

This Review has discussed numerous NP designs optimized for therapeutic delivery and engineered to overcome the heterogeneous biological barriers found across patient populations and diseases. These barriers to delivery are complicated by patient comorbidities, varying stages of disease progression and unique physiologies. This diverse array of needs can be met using NPs designed for different patient populations or pathologies, or intersections of the two. NP platforms offer an assortment of modifiable features such as size, shape, charge, surface properties and responsiveness that can be selected to optimize delivery for a specific application, therapeutic and patient population. This customization can be utilized synergistically with precision medicine therapies to improve patient stratification methods when screening NP platforms, widen the accessibility of precision therapeutics by allowing new patients to qualify for existing therapies with newly enhanced delivery mechanisms and, ultimately, increase the overall therapeutic efficacy of both precision medicines and NP delivery platforms.

Of these NP characteristics, size and shape have been extensively studied across numerous biological states, and, in some cases, trends have been identified that can be used for intelligent NP design. For example, NP charge is of particular importance in muco-penetrating applications and intracellular applications that require endosomal escape, whereas targeting surface markers takes precedent in applications where specific cell types must uptake NPs, as in many cancer and immunotherapy applications. However, as the design considerations become more complicated, so do efforts to generalize trends across large populations - sacrificing the accuracy of the findings within a small population in the hope of generating an all-encompassing principle of delivery. Therefore, investigations of NP design and the resulting interactions within the human body need to be more thoroughly analysed to improve the specificity of these claims, especially as we move towards stratifying patient populations to determine the most suitable NP platforms for these subgroups. Through the continued exploration of NP technologies in laboratory settings, researchers have the opportunity to collect data and analyse outcomes to add to the ever-growing library of 
known design-function relationship trends in nanomedicine. However, it is imperative that the trends observed in research settings be contextualized before attempting to generalize findings broadly, as seemingly minor differences in NP composition, animal models and pathology may greatly alter the performance of NPs and must be considered when moving NP technology towards clinical translation.

Current clinical successes with NPs in precision medicine have been largely diagnostic, such as the ability to recognize early stages of a disease by specific ligandreceptor interactions or the use of biomarkers to identify which therapeutics might be best for a particular patient. For example, determining the level of the EPR effect that a cancer patient exhibits can inform how effectively a NP therapy would accumulate at the solid tumour site ${ }^{152}$. However, this Review has focused on potential therapeutic applications of NPs - specifically, their use in the precision medicine fields of oncology, immunotherapy and genome engineering - as these platforms have immense potential to improve efficacy of precision medicine therapies but have yet to see the clinical progress achieved by diagnostic applications. This lack of clinical progress is likely because NP platforms are screened for efficacy in broad populations, in which the vast heterogeneity of biological barriers in patients could mask the potential for successfully treating slightly smaller subgroups. As mentioned previously, the introduction of stratified patient populations may also accelerate clinical progress, as stratified populations will likely respond more uniformly to NP treatment. However, as ongoing clinical trials are generally unstratified, we are currently unable to predict which NP platforms will be most useful for precision applications, and more stratified trials are necessary.

Of course, moving to screen NPs through a precision lens - thus, limiting the number of patients that are eligible to receive a medication - will reduce the potential market size of each NP-based therapeutic. This reduction may raise concern when considering the high cost of development for advanced NP designs and, thus, the increased financial risks associated with the potentially failed clinical translation of a NP formulation. However, NP platforms that are found to work well in specific patient populations may have applications in the delivery of numerous therapeutics, both precision-based and generic. Thus, the development of one highly effective NP platform for a stratified group could lead to multiple successful clinical applications. Furthermore, precision
NP designs may allow for greater therapeutic efficacy compared with NPs developed for broad populations, and significant improvements in survival, quality of life and even dosing could justify the higher price point of these precision delivery systems.

As more advanced NP designs are explored, this research could influence the future rational design of drug carriers for various therapeutics, both personalized and generic, thereby benefitting an array of cargos including small molecules, nucleotides and proteins. Furthermore, the approval of more NP platforms could ease the path to the clinic for novel applications of these NPs for the delivery of previously approved therapeutic cargos. By working to develop more NP platforms and precision medicines for FDA approval and clinical use, we are taking steps towards more modular patient therapy designs and creating the potential for future prescriptions to not just include the optimal therapy but also pair it with the optimized delivery platform. The concept of offering multiple delivery platforms for a single therapeutic is not new in the clinical marketplace. Commonly prescribed drugs such as those used for birth control are already offered in multiple forms (oral pills, injections and implants) to fit the patient's lifestyle. The expansion of both precision medicine and advanced NP platforms will contribute to the continued clinical progress of personalized medicines, allowing for seemingly niche markets to grow.

In precision medicine-relevant applications, the usage of NPs allows for improved cellular targeting, fewer off-target effects and more tailored therapies such as multidrug treatments. All of this can be achieved by engineering NPs for the application at hand, improving accumulation at the site of interest and introducing responsivity for on-demand drug release, to minimize unwanted toxicities and enable a new range of dosages or combinatorial treatments. By optimizing this specificity and local activity of NP delivery systems, the effects of precision medicine therapeutics can be improved as well, widening the populations they benefit and improving patient outcome overall. As the work described in this Review shows, intelligent NP design can improve precision medicine as a whole and the insight provided by precision medicine - such as patient stratification and genetic profiling - can inform the rational selection of a NP platform to, ultimately, generate the ideal NP-based precision therapy.

Published online 4 December 2020
1. The White House Office of the Press Secretary. National Nanotechnology Initiative: leading to the next industrial revolution. White House https:/ clintonwhitehouse 4.archives.gov/WH/New/html/ 20000121_4.html (2000)

2. Kou, L. et al. Transporter-guided delivery of nanoparticles to improve drug permeation across cellular barriers and drug exposure to selective cell types. Front. Pharmacol. 9, 1-16 (2018).

3. Blanco, E., Shen, H. \& Ferrari, M. Principles of nanoparticle design for overcoming biological barriers to drug delivery. Nat. Biotechnol. 33, 941-951 (2015)

4. Mitragotri, S. et al. Drug delivery research for the future: expanding the nano horizons and beyond. J. Control. Release 246, 183-184 (2017).

5. Wechsler, M. E., Vela Ramirez, J. E. \& Peppas, N. A. 110th anniversary: nanoparticle mediated drug delivery for the treatment of Alzheimer's disease: crossing the blood-brain barrier. Ind. Eng. Chem. Res 58, 15079-15087 (2019).

6. Hua, S., de Matos, M. B. C., Metselaar, J. M. $\&$ Storm, G. Current trends and challenges in the clinical translation of nanoparticulate nanomedicines: pathways for translational development and commercialization. Front. Pharmacol. 9, 790 (2018).

7. Anselmo, A. C. \& Mitragotri, S. Nanoparticles in the clinic: an update. Bioeng. Transl. Med. 4, 1-16 (2019).

This review comprehensively examines recent NP systems that are currently clinically approved or under clinical trials

8. Wagner, A. M., Gran, M. P. \& Peppas, N. A Designing the new generation of intelligent biocompatible carriers for protein and peptide delivery. Acta Pharm. Sin. B 8, 147-164 (2018).

9. Culver, H. R., Clegg, J. R. \& Peppas, N. A. Analyteresponsive hydrogels: intelligent materials for biosensing and drug delivery. Acc. Chem. Res. 50, 170-178 (2017)

10. Ruan, S. et al. Matrix metalloproteinase-sensitive size-shrinkable nanoparticles for deep tumor penetration and $\mathrm{pH}$ triggered doxorubicin release. Biomaterials 60, 100-110 (2015).

11. Clegg, J. R. et al. Synthetic networks with tunable responsiveness, biodegradation, and molecular recognition for precision medicine applications. Sci. Adv. 5, eaax7946 (2019).

12. Cheng, $Q$. et al. Selective organ targeting (SORT) nanoparticles for tissue-specific mRNA delivery and 
CRISPR-Cas gene editing. Nat. Nanotechnol. 15, 313-320 (2020)

13. Collins, F. S. \& Varmus, H. A new initiative on precision medicine. N. Engl. J. Med. 372, 793-795 (2015)

14. Fonseca-Santos, B., Gremião, M. P. D. \& Chorilli, M Nanotechnology-based drug delivery systems for the treatment of Alzheimer's disease. Int. J. Nanomed. 10 4981-5003 (2015)

15. Sercombe, L. et al. Advances and challenges of liposome assisted drug delivery. Front. Pharmacol. 6, 286 (2015).

16. Fenton, O. S., Olafson, K. N., Pillai, P. S., Mitchell, M. J. \& Langer, R. Advances in biomaterials for drug delivery. Adv. Mater. 30, 1705328 (2018).

17. Sarfraz, M. et al. Development of dual drug loaded nanosized liposomal formulation by a reengineered ethanolic injection method and its pre-clinical pharmacokinetic studies. Pharmaceutics 10, 1-22 (2018).

18. Sedighi, M. et al. Rapid optimization of liposome characteristics using a combined microfluidics and design-of-experiment approach. Drug Deliv. Transl. Res. 9, 404-413 (2019).

19. Alyautdin, R., Khalin, I., Nafeeza, M. I., Haron, M. H $\&$ Kuznetsov, D. Nanoscale drug delivery systems and the blood-brain barrier. Int. J. Nanomed. 9, 795-811 (2014).

20. Leung, A. K. K., Tam, Y. Y. C., Chen, S., Hafez, I. M. $\&$ Cullis, P. R. Microfluidic mixing: a general method for encapsulating macromolecules in lipid nanoparticle systems. J. Phys. Chem. B 119, 8698-8706 (2015).

21. Cheng, X. \& Lee, R. J. The role of helper lipids in lipid nanoparticles (LNPs) designed for oligonucleotide delivery. Adv. Drug Deliv. Rev. 99, 129-137 (2016).

22. Kulkarni, J. A., Witzigmann, D., Leung, J., Tam, Y. Y. C. $\&$ Cullis, P. R. On the role of helper lipids in lipid nanoparticle formulations of siRNA. Nanoscale 11 21733-21739 (2019).

23. Berraondo, P., Martini, P. G. V., Avila, M. A. $\&$ Fontanellas, A. Messenger RNA therapy for rare genetic metabolic diseases. Gut 68, 1323-1330 (2019).

24. Vhora, I., Lalani, R., Bhatt, P., Patil, S. \& Misra, A Lipid-nucleic acid nanoparticles of novel ionizable lipids for systemic BMP-9 gene delivery to bonemarrow mesenchymal stem cells for osteoinduction. Int. J. Pharm. 563, 324-336 (2019).

25. Patel, S., Ryals, R. C., Weller, K. K., Pennesi, M. E. $\&$ Sahay, G. Lipid nanoparticles for delivery of messenger RNA to the back of the eye. J. Control. Rel. 303, 91-100 (2019).

26. Brown, S. B., Wang, L., Jungels, R. R. \& Sharma, B. Effects of cartilage-targeting moieties on nanoparticle biodistribution in healthy and osteoarthritic joints. Acta Biomater. 101, 469-483 (2019).

27. Zhang, C. X. et al. Mitochondria-targeted cyclosporin A delivery system to treat myocardial ischemia reperfusion injury of rats. J. Nanobiotechnol. 17 , $1-16$ (2019)

28. Le, Z. et al. Hydrogen-bonded tannic acid-based anticancer nanoparticle for enhancement of ora chemotherapy. ACS Appl. Mater. Interfaces 10 , 42186-42197 (2018).

29. He, C. et al. siRNA release kinetics from polymeric nanoparticles correlate with RNAi efficiency and inflammation therapy via oral delivery. Acta Biomater. 103, 213-222 (2020).

30. Zhang, L. et al. Microfluidic-assisted polymer-protein assembly to fabricate homogeneous functional nanoparticles. Mater. Sci. Eng. C 111, 110768 (2020).

31. Caldorera-Moore, M., Vela Ramirez, J. E. \& Peppas, N. A. Transport and delivery of interferon-a through epithelial tight junctions via $\mathrm{pH}$-responsive poly(methacrylic acid-grafted-ethylene glycol) nanoparticles. J. Drug Target. 27, 582-589 (2019).

32. Knight, F. C. et al. Mucosal immunization with a pH-responsive nanoparticle vaccine induces protective CD8+ lung-resident memory T cells. ACS Nano 13, 10939-10960 (2019).

33. Strand, M. S. et al. Precision delivery of RAS-inhibiting SiRNA to KRAS driven cancer via peptide-based nanoparticles. Oncotarget 10, 4761-4775 (2019).

34. Jose, S. et al. Transferrin-conjugated docetaxel-PLGA nanoparticles for tumor targeting: influence on MCF-7 cell cycle. Polymers 11, 1905 (2019).

35. Liu, X. et al. Glucose and $\mathrm{H}_{2} \mathrm{O}_{2}$ dual-responsive polymeric micelles for the self-regulated release of insulin. ACS Appl. Bio Mater. 3, 1598-1606 (2020).

36. Afsharzadeh, M., Hashemi, M., Mokhtarzadeh, A., Abnous, K. ¿ Ramezani, M. Recent advances in co-delivery systems based on polymeric nanoparticle for cancer treatment. Artif. Cells Nanomed. Biotechnol. 46, 1095-1110 (2018)

37. Patra, J. K. et al. Nano based drug delivery systems: recent developments and future prospects. J. Nanobiotechnol. 16, 71 (2018).

38. Volpatti, L. R. et al. Glucose-responsive nanoparticles for rapid and extended self-regulated insulin delivery. ACS Nano 14, 488-497 (2020).

39. Rideau, E., Dimova, R., Schwille, P., Wurm, F. R. $\&$ Landfester, K. Liposomes and polymersomes: a comparative review towards cell mimicking. Chem. Soc. Rev. 47, 8572-8610 (2018).

40. Shae, D. et al. Endosomolytic polymersomes increase the activity of cyclic dinucleotide STING agonists to enhance cancer immunotherapy. Nat. Nanotechnol. 14, 269-278 (2019).

41. Zelmer, C. et al. Organelle-specific targeting of polymersomes into the cell nucleus. Proc. Natl Acad. Sci. USA 117, 2770-2778 (2020).

42. Lee, S.-W. et al. An open-label, randomized, parallel, phase II trial to evaluate the efficacy and safety of a cremophor-free polymeric micelle formulation of paclitaxel as first-line treatment for ovarian cancer: a Korean Gynecologic Oncology Group Study (KGOG-3021). Cancer Res. Treat. 50, 195-203 (2018).

43. Xu, L., Zhang, H. \& Wu, Y. Dendrimer advances for the central nervous system delivery of therapeutics. ACS Chem. Neurosci. 5, 2-13 (2014).

44. Mendes, L. P., Pan, J. \& Torchilin, V. P. Dendrimers as nanocarriers for nucleic acid and drug delivery in cancer therapy. Molecules 22, 1401 (2017).

45. Kannan, R. M., Nance, E., Kannan, S. \& Tomalia, D. A Emerging concepts in dendrimer-based nanomedicine from design principles to clinical applications. J. Intern Med. 276, 579-617 (2014).

46. Menjoge, A. R., Kannan, R. M \& Tomalia, D. A Dendrimer-based drug and imaging conjugates: design considerations for nanomedical applications. Drug Discov. Today 15, 171-185 (2010).

47. Cao, S.-J. et al. Nanoparticles: oral delivery for protein and peptide drugs. AAPS PharmSciTech 20, 190 (2019).

48. Tian, H. et al. Uniform core-shell nanoparticles with thiolated hyaluronic acid coating to enhance oral delivery of insulin. Adv. Healthc. Mater. 7, 1-12 (2018).

49. Valcourt, D. M., Dang, M. N., Scully, M. A. \& Day, E. S. Nanoparticle-mediated co-delivery of Notch-1 antibodies and ABT-737 as a potent treatment strategy for triple-negative breast cancer. ACS Nano 14, 3378-3388 (2020).

50. Yang, W., Liang, H., Ma, S., Wang, D. \& Huang, J. Gold nanoparticle based photothermal therapy: development and application for effective cancer treatment. Sustain. Mater. Technol. 22, e00109 (2019).

51. Wang, J., Potocny, A. M., Rosenthal, J. \& Day, E. S Gold nanoshell-linear tetrapyrrole conjugates for near infrared-activated dual photodynamic and photothermal therapies. ACS Omega 5, 926-940 (2020).

52. Bobo, D., Robinson, K. J., Islam, J., Thurecht, K. J. \& Corrie, S. R. Nanoparticle-based medicines: a review of FDA-approved materials and clinical trials to date Pharm. Res. 33, 2373-2387 (2016).

53. Arias, L. S. et al. Iron oxide nanoparticles for biomedical applications: a perspective on synthesis, drugs, antimicrobial activity, and toxicity. Antibiotics 7, 46 (2018)

54. Huang, K.-W. et al. Highly efficient and tumor-selective nanoparticles for dual-targeted immunogene therapy against cancer. Sci. Adv. 6, eaax5032 (2020).

55. Xu, C., Nam, J., Hong, H., Xu, Y. \& Moon, J. J. Positron emission tomography-guided photodynamic therapy with biodegradable mesoporous silica nanoparticles for personalized cancer immunotherapy. ACS Nano 13, 12148-12161 (2019).

56. Wagner, A. M., Knipe, J. M., Orive, G. \& Peppas, N. A Quantum dots in biomedical applications. Acta Biomater. 94, 44-63 (2019).

57. Zhang, Y., Meng, S., Ding, J., Peng, Q. \& Yu, Y. Transition metal-coordinated graphitic carbon nitride dots as a sensitive and facile fluorescent probe for $\beta$-amyloid peptide detection. Analyst 144, 504-51 (2019).

58. Manshian, B. B., Jiménez, J., Himmelreich, U. \& Soenen, S. J. Personalized medicine and follow-up of therapeutic delivery through exploitation of quantum dot toxicity. Biomaterials 127, 1-12 (2017)

59. Aguado, B. A., Grim, J. C., Rosales, A. M., Watson-Capps, J. J. \& Anseth, K. S. Engineering precision biomaterials for personalized medicine. Sci. Transl. Med. 10, 8645 (2018).

60. van der Meel, R. et al. Smart cancer nanomedicine. Nat. Nanotechnol. 14, 1007-1017 (2019).

61. Scheetz, L. et al. Engineering patient-specific cancer immunotherapies. Nat. Biomed. Eng. 3, 768-782 (2019).

62. Papi, M. et al. Converting the personalized biomolecular corona of graphene oxide nanoflakes into a high-throughput diagnostic test for early cancer detection. Nanoscale 11, 15339-15346 (2019).

63. Shaban, M., Hasanzadeh, M. \& Solhi, E. An $\mathrm{Fe}_{3} \mathrm{O}$ PEDOT:PSS nanocomposite as an advanced electroconductive material for the biosensing of the prostate-specific antigen in unprocessed human plasma samples. Anal. Methods 11, 5661-5672 (2019).

64. Zhang, T. et al. The synergistic effect of Au-COF nanosheets and artificial peroxidase Au@ZIF-8(NiPd) rhombic dodecahedra for signal amplification for biomarker detection. Nanoscale 11, 20221-20227 (2019).

65. Kaur, A Shimoni, O \& Wallach, M. Novel screening test for celiac disease using peptide functionalised gold nanoparticles. World J. Gastroenterol. 24 5379-5390 (2018).

66. Tan, T. et al. Bioinspired lipoproteins-mediated photothermia remodels tumor stroma to improve cancer cell accessibility of second nanoparticles. Nat. Commun. 10, 3322 (2019).

67. Wilson, R. A. et al. MicroRNA regulation of endothelia TREX1 reprograms the tumour microenvironment. Nat. Commun. 7, 13597 (2016)

68. Papa, A. L. et al. Ultrasound-sensitive nanoparticle aggregates for targeted drug delivery. Biomaterials 139, 187-194 (2017).

69. Chen, Q. et al. Photothermal therapy promotes tumor infiltration and antitumor activity of CAR T cells. Adv. Mater. 31, e1900192 (2019).

70. Patel, R. B. et al. Development of an in situ cancer vaccine via combinational radiation and bacterialmembrane-coated nanoparticles. Adv. Mater. 31 e1902626 (2019).

71. Wilhelm, S. et al. Analysis of nanoparticle delivery to tumours. Nat. Rev. Mater. 1, 16014 (2016). This comprehensive analysis of NP delivery surveys literature from 2005 to 2015 and finds that, on average, only $0.7 \%$ of the administered NP dose is delivered to a solid tumour, and, further, explores possible reasons for this inefficiency.

72. Ensign, L. M., Cone, R. \& Hanes, J. Oral drug delivery with polymeric nanoparticles: the gastrointestinal mucus barriers. Adv. Drug Deliv. Rev. 64, 557-570 (2012).

This review thoroughly examines barriers of the gastrointestinal tract and more deeply explores their effects on orally administered NPs.

73. Nag, O. K. \& Delehanty, J. B. Active cellular and subcellular targeting of nanoparticles for drug delivery. Pharmaceutics 11, 543 (2019).

74. Oliva, N. et al. Regulation of dendrimer/dextran material performance by altered tissue microenvironment in inflammation and neoplasia. Sci. Transl. Med. 7, 272 ra11 (2015).

This study looks at the impact of disease on the tissue microenvironment and discovers that in states of carcinogenesis and inflammation the material-tissue interactions change, even when established biomaterials are used.

75. Gehr, P. \& Zellner, R. Biological Responsese to Nanoscale Particles: Molecular and Cellular Aspects and Methodological Approaches (Springer, 2019).

76. Hoshyar, N., Gray, S., Han, H. \& Bao, G. The effect of nanoparticle size on in vivo pharmacokinetics and cellular interaction. Nanomedicine 11, 673-692 (2016).

This review presents the influence of NP size on cellular uptake, biodistribution and pharmacokinetics, and also introduces models for interpretation of this result.

77. Wang, X. Y., Ishida, T. \& Kiwada, H. Anti-PEG IgM elicited by injection of liposomes is involved in the enhanced blood clearance of a subsequent dose of PEGylated liposomes. J. Control. Rel. 119, 236-244 (2007).

78. McSweeney, M. D. et al. A minimal physiologically based pharmacokinetic model that predicts anti-PEC IgG-mediated clearance of PEGylated drugs in human and mouse. J. Control. Rel. 284, 171-178 (2018).

79. Yang, Q. et al. Analysis of pre-existing IgG and IgM antibodies against polyethylene glycol (PEG) in the general population. Anal. Chem. 88, 11804-11812 (2016). 
80. Chen, B. M. et al. Measurement of pre-existing IgC and IgM antibodies against polyethylene glycol in healthy individuals. Anal. Chem. 88, 10661-10666 (2016).

81. Hu, C. M. J. et al. Nanoparticle biointerfacing by platelet membrane cloaking. Nature 526, 118-121 (2015).

82. Wibroe, P. P. et al. Bypassing adverse injection reactions to nanoparticles through shape modification and attachment to erythrocytes. Nat. Nanotechnol. 12, 589-594 (2017).

83. Anselmo, A. C. et al. Platelet-like nanoparticles: mimicking shape, flexibility, and surface biology of platelets to target vascular injuries. ACS Nano 8 , 11243-11253 (2014)

84. Palanikumar, L. et al. pH-responsive high stability polymeric nanoparticles for targeted delivery of anticancer therapeutics. Commun. Biol. 3, 95 (2020).

85. Tanaka, R. et al. Furry nanoparticles: synthesis and characterization of nanoemulsion-mediated core crosslinked nanoparticles and their robust stability in vivo. Polym. Chem. 11, 4408-4416 (2020).

86. Jarvis, M. et al. Detachment of ligands from nanoparticle surface under flow and endothelial cell contact: assessment using microfluidic devices. Bioeng. Transl. Med. 3, 148-155 (2018).

87. Hosta-Rigau, L. \& Städler, B. Shear stress and its effect on the interaction of myoblast cells with nanosized drug delivery vehicles. Mol. Pharm. 10, 2707-2712 (2013)

88. Khor, S. Y. et al. Elucidating the influences of size, surface chemistry, and dynamic flow on cellular association of nanoparticles made by polymerizationinduced self-assembly. Small 14, 1-13 (2018).

89. Cooley, M. et al. Influence of particle size and shape on their margination and wall-adhesion: implications in drug delivery vehicle design across nano-to-micro scale. Nanoscale 10, 15350-15364 (2018).

90. Pieter, S. \& Mahmoudi, M. Drug Delivery Systems (World Scientific, 2017)

91. Da Silva-Candal, A. et al. Shape effect in active targeting of nanoparticles to inflamed cerebral endothelium under static and flow conditions. J. Control. Rel. 309, 94-105 (2019). This work studies the effect of shape on NP targeting efficiency in the bloodstream, with rod-shaped particles showing better accumulation than spherical.

92. Uhl, C. G., Gao, Y., Zhou, S. \& Liu, Y. The shape effect on polymer nanoparticle transport in a blood vessel. RSC Adv. 8, 8089-8100 (2018).

93. Malota, Z., Glowacki, J., Sadowski, W. \& Kostur, M. Numerical analysis of the impact of flow rate, heart rate, vessel geometry, and degree of stenosis on coronary hemodynamic indices. BMC CardiovasC. Disord. 18, 1-16 (2018).

94. Chacón-Lozsăn, F., et al. Hemodynamic management of high blood pressure. Ther. Adv. Cardiol. 1, 73-79 (2017).

95. Tenzer, S. et al. Rapid formation of plasma protein corona critically affects nanoparticle pathophysiology. Nat. Nanotechnol. 8, 772-781 (2013).

96. Cox, A. et al. Evolution of nanoparticle protein corona across the blood-brain barrier. ACS Nano 12 , 7292-7300 (2018)

97. Schwartz, S. Unmet needs in developing nanoparticles for precision medicine. Nanomedicine 12, 271-274 (2017).

98. von Roemeling, C., Jiang, W., Chan, C. K Weissman, I. L. \& Kim, B. Y. S. Breaking down the barriers to precision cancer nanomedicine. Trends Biotechnol. 35, 159-171 (2017).

99. Chen, D., Parayath, N., Ganesh, S., Wang, W. $\Sigma$ Amiji, M. The role of apolipoprotein- and vitronectin-enriched protein corona on lipid nanoparticles for vivo targeted delivery and transfection of oligonucleotides in murine tumor models. Nanoscale 11, 18806-18824 (2019). This study analyses the impact of NP make-up on protein corona and resulting tumour-specific delivery, showing that PEG content can influence outcome and highlighting the ability of NP design features to dictate biodistribution.

100. Fornaguera, C. et al. In vivo retargeting of poly( $\beta$ aminoester) (OM-PBAE) nanoparticles is influenced by protein corona. Adv. Healthc. Mater. 8, 1-11 (2019).

101. Bertrand, N. et al. Mechanistic understanding of in vivo protein corona formation on polymeric nanoparticles and impact on pharmacokinetics. Nat. Commun. 8, 777 (2017)

102. Yeo, E. L. L., Cheah, J. U. J., Thong, P. S. P., Soo, K. C. \& Kah, J. C.Y. Gold nanorods coated with apolipoprotein $\mathrm{E}$ protein corona for drug delivery. ACS Appl. Nano Mater. 2, 6220-6229 (2019).

103. Dal Magro, R. et al. ApoE-modified solid lipid nanoparticles: a feasible strategy to cross the bloodbrain barrier. J. Control. Rel. 249, 103-110 (2017)

104. Dong, Y. et al. Lipopeptide nanoparticles for potent and selective siRNA delivery in rodents and nonhuman primates. Proc. Natl Acad. Sci. USA 111, 3955-3960 (2014).

105. Key, J. et al. Soft discoidal polymeric nanoconstructs resist macrophage uptake and enhance vascular targeting in tumors. ACS Nano 9, 11628-11641 (2015).

106. Hui, Y. et al. Role of nanoparticle mechanical properties in cancer drug delivery. ACS Nano 13. 7410-7424 (2019).

107. Nizzero, S., Ziemys, A. \& Ferrari, M. Transport barriers and oncophysics in cancer treatment. Trends Cancer $\mathbf{4}$ 277-280 (2018)

108. Kumar, S., Anselmo, A. C., Banerjee, A., Zakrewsky, M $\&$ Mitragotri, S. Shape and size-dependent immune response to antigen-carrying nanoparticles. J. Control. Rel. 220, 141-148 (2015).

109. Xie, X., Liao, J., Shao, X., Li, Q. \& Lin, Y. The effect of shape on cellular uptake of gold nanoparticles in the forms of stars, rods, and triangles. Sci. Rep. 7, 1-9 (2017).

110. Wang, W., Gaus, K., Tilley, R. D. \& Gooding, J. J. The impact of nanoparticle shape on cellular internalisation and transport: what do the different analysis methods tell us? Mater. Horiz. 6 , 1538-1547 (2019).

111. Garapaty, A. \& Champion, J. A. Shape of ligand immobilized particles dominates and amplifies the macrophage cytokine response to ligands. PLoS One 14, 12-14 (2019)

112. Ganson, N. J. et al. Pre-existing anti-polyethylene glycol antibody linked to first-exposure allergic reactions to pegnivacogin, a PEGylated RNA aptamer J. Allergy Clin. Immunol. 137, 1610-1613.e7 (2016)

113. Povsic, T. J. et al. Pre-existing anti-PEG antibodies are associated with severe immediate allergic reactions to pegnivacogin, a PEGylated aptamer. J. Allergy Clin. Immunol. 138, 1712-1715 (2016)

114. Zhong, Q., Merkel, O. M., Reineke, J. J. $\Sigma$ da Rocha, S. R. P. Effect of the route of administration and PEGylation of poly(amidoamine) dendrimers on their systemic and lung cellular biodistribution. Mol. Pharm. 13, 1866-1878 (2016)

115. Battaglia, L. et al. Lipid nanoparticles for intranasal administration: application to nose-to-brain delivery. Expert Opin. Drug Deliv. 15, 369-378 (2018).

116. Dölen, Y. et al. Nanovaccine administration route is critical to obtain pertinent iNKt cell help for robust anti-tumor T and B cell responses. Oncoimmunology 9, 1738813 (2020).

117. McLennan, D. N., Porter, C. J. H. \& Charman, S. A. Subcutaneous drug delivery and the role of the lymphatics. Drug Discov. Today Technol. 2, 89-96 (2005).

118. Dong, W. et al. Comparative study of mucoadhesive and mucus-penetrative nanoparticles based on phospholipid complex to overcome the mucus barrier for inhaled delivery of baicalein. Acta Pharm. Sin. B 10, 1576-1585 (2019)

119. Cone, R. A. Barrier properties of mucus. Adv. Drug Deliv. Rev. 61, 75-85 (2009).

120. Wu, L. et al. Quantitative comparison of three widelyused pulmonary administration methods in vivo with radiolabeled inhalable nanoparticles. Eur. J. Pharm. Biopharm. 152, 108-115 (2020).

This study investigates three widely used routes of pulmonary administration in mouse models, revealing that the administration route affects polymeric NP biodistribution and highlighting another means of heterogenizing NP delivery.

121. Pant, K. et al. Active targeting of dendritic polyglycerols for diagnostic cancer imaging. Small 16, 1905013. (2019).

122. Yi, Y. et al. Glucose-linked sub-50-nm unimer polyion complex-assembled gold nanoparticles for targeted siRNA delivery to glucose transporter 1-overexpressing breast cancer stem-like cells. J. Control. Release 295 , 268-277 (2019)

123. Yan, Y. et al. Dually active targeting nanomedicines based on a direct conjugate of two purely natural ligands for potent chemotherapy of ovarian tumors. ACS Appl. Mater. Interfaces 11, 46548-46557 (2019).

124. Ahmad, A., Khan, F., Mishra, R. K. \& Khan, R. Precision cancer nanotherapy: evolving role of multifunctional nanoparticles for cancer active targeting. J. Med. Chem. 62, 10475-10496 (2019).
125. Du, Y., Liu, X., Liang, Q., Liang, X. J. \& Tian, J. Optimization and design of magnetic ferrite nanoparticles with uniform tumor distribution for highly sensitive MRI/MPI performance and improved magnetic hyperthermia therapy. Nano Lett. 19 3618-3626 (2019).

126. Hong, S. T. et al. Improving the anticancer effect of afatinib and microRNA by using lipid polymeric nanoparticles conjugated with dual $\mathrm{pH}$-responsive and targeting peptides. J. Nanobiotechnol. 17, 89 (2019).

127. Saraiva, C. et al. Nanoparticle-mediated brain drug delivery: overcoming blood-brain barrier to treat neurodegenerative diseases. J. Control. Release $\mathbf{2 3 5}$, 34-47 (2016)

128. Zhou, Q. et al. Enzyme-activatable polymer-drug conjugate augments tumour penetration and treatment efficacy. Nat. Nanotechnol. 14, 799-809 (2019).

129. Sindhwani, S. et al. The entry of nanoparticles into solid tumours. Nat. Mater. 19, 566-575 (2020). This groundbreaking study shows that interendothelial gaps are not responsible for NP entry into solid tumours as was previously thought, but instead finds that up to $97 \%$ of NPs actively enter tumours through endothelial cells.

130. Johnsen, K. B. et al. Modulating the antibody density changes the uptake and transport at the blood-brain barrier of both transferrin receptor-targeted gold nanoparticles and liposomal cargo. J. Control. Release 295, 237-249 (2019).

131. Sousa, F., Dhaliwal, H. K., Gattacceca, F., Sarmento, B. \& Amiji, M. M. Enhanced anti-angiogenic effects of bevacizumab in glioblastoma treatment upon intranasal administration in polymeric nanoparticles. J. Control. Release 309, 37-47 (2019).

132. Musumeci, T., Bonaccorso, A. \& Puglisi, G. Epilepsy disease and nose-to-brain delivery of polymeric nanoparticles: an overview. Pharmaceutics 11, 118 (2019).

133. Bruinsmann, F. A. et al. Nasal drug delivery of anticancer drugs for the treatment of glioblastoma: preclinical and clinical trials. Molecules 24, 4312 (2019).

134. Lamson, N. G., Berger, A., Fein, K. C. $\&$ Whitehead, K. A. Anionic nanoparticles enable the oral delivery of proteins by enhancing intestinal permeability. Nat. Biomed. Eng. 4, 84-96 (2020). This work screens inorganic NPs for the oral delivery of proteins and finds the properties needed to enhance intestinal permeation via opening tight junctions, which include negative charge and small size.

135. Banerjee, A., Qi, J., Gogoi, R., Wong, J. \& Mitragotri, S. Role of nanoparticle size, shape and surface chemistry in oral drug delivery. J. Control. Release $\mathbf{2 3 8}$ 176-185 (2016)

136. Yong, J. M., Mantaj, J., Cheng, Y. \& Vllasaliu, D. Delivery of nanoparticles across the intestinal epithelium via the transferrin transport pathway. Pharmaceutics 11, 298 (2019).

137. Zhang, S. et al. BSA nanoparticles modified with $\mathrm{N}$-acetylcysteine for improving the stability and mucoadhesion of curcumin in the gastrointestinal tract. J. Agric. Food Chem. 67, 9371-9381 (2019).

138. Zheng, N. et al. Mesoporous silica nanorods for improved oral drug absorption. Artif. Cells Nanomed. Biotechnol. 46, 1132-1140 (2018).

139. Li, D. et al. Influence of particle geometry on gastrointestinal transit and absorption following oral administration. ACS Appl. Mater. Interfaces $\mathbf{9}$, 42492-42502 (2017).

140. Zhuang, J. et al. The influence of nanoparticle shape on bilateral exocytosis from Caco-2 cells. Chin. Chem. Lett. 29, 1815-1818 (2018).

141. Berardi, A. \& Baldelli Bombelli, F. Oral delivery of nanoparticles - let's not forget about the protein corona. Expert Opin. Drug Deliv. 16, 563-566 (2019).

142. Durăn-Lobato, M., Niu, Z. \& Alonso, M. J. Oral delivery of biologics for precision medicine. Adv. Mater. 32, e1901935 (2019)

143. Kruse, C. R. et al. The effect of $\mathrm{pH}$ on cell viability, cell migration, cell proliferation, wound closure, and wound reepithelialization: In vitro and in vivo study. Wound Repair Regen. 25, 260-269 (2017).

144. Hathaway, H. et al. Thermally triggered release of the bacteriophage endolysin CHAPK and the bacteriocin lysostaphin for the control of methicillin resistant Staphylococcus aureus (MRSA). J. Control. Release 245, 108-115 (2017)

145. Holme, M. N. et al. Shear-stress sensitive lenticular vesicles for targeted drug delivery. Nat. Nanotechnol. 7, 536-543 (2012). 
146. Sykes, E. A. et al. Tailoring nanoparticle designs to target cancer based on tumor pathophysiology. Proc. Natl Acad. Sci. USA 113, E1142-E1151 (2016).

147. Zan, Y., Dai, Z., Liang, L., Deng, Y. \& Dong, L. Co-delivery of plantamajoside and sorafenib by a multi-functional nanoparticle to combat the drug resistance of hepatocellular carcinoma through reprograming the tumor hypoxic microenvironment. Drug Deliv. 26, 1080-1091 (2019).

This work focuses on a NP system that can reprogramme the tumour microenvironment, working to reverse drug resistance of tumour cells and resensitize patients to existing first-line therapies.

148. Dai, Q. et al. Quantifying the ligand-coated nanoparticle delivery to cancer cells in solid tumors ACS Nano 12, 8423-8435 (2018).

149. Witten, J. \& Ribbeck, K. The particle in the spider's web: transport through biological hydrogels. Nanoscale 9, 8080-8095 (2017)

150. Nagel, G., Sousa-Herves, A., Wedepohl, S. ¿ Calderón, M. Matrix metalloproteinase-sensitive multistage nanogels promote drug transport in 3D tumor model. Theranostics 10, 91-108 (2020).

151. Price, L. S. L., Stern, S. T., Deal, A. M., Kabanov, A. V. $\&$ Zamboni, W. C. A reanalysis of nanoparticle tumo delivery using classical pharmacokinetic metrics. Sci. Adv. 6, eaay9249 (2020)

152. Lee, H. et al. ${ }^{64} \mathrm{Cu}-\mathrm{MM}-302$ positron emission tomography quantifies variability of enhanced permeability and retention of nanoparticles in relation to treatment response in patients with metastatic breast cancer. Clin. Cancer Res. 23, 4190-4202 (2017)

153. Ding, J. et al. Engineered nanomedicines with enhanced tumor penetration. Nano Today 29 100800 (2019).

154. Wadajkar, A. S. et al. Decreased non-specific adhesivity, receptor targeted (DART) nanoparticles exhibit improved dispersion, cellular uptake, and tumor retention in invasive gliomas. J. Control. Release 267, 144-153 (2017).

155. Nance, E. A. et al. A dense poly(ethylene glycol) coating improves penetration of large polymeric nanoparticles within brain tissue. Sci. Transl. Med. 4 149ra119 (2012)

156. Stephen, Z. R. et al. Time-resolved MRI assessment of convection-enhanced delivery by targeted and nontargeted nanoparticles in a human glioblastoma mouse model. Cancer Res. 79, 4776-4786 (2019).

157. Fahy, J. V. \& Dickey, B. F. Airway mucus function and dysfunction. N. Engl. J. Med. 363, 2233-2247 (2010)

158. Livraghi-Butrico, A. et al. Contribution of mucus concentration and secreted mucins Muc5ac and Muc5 $b$ to the pathogenesis of muco-obstructive lung disease. Mucosal Immunol. 10, 395-407 (2017).

159. Ho, L. W. C., Liu, Y., Han, R., Bai, O. \& Choi, C. H. J. Nano-cell interactions of non-cationic bionanomaterials. Acc. Chem. Res. 52, 1519-1530 (2019)

160. Behzadi, S. et al. Cellular uptake of nanoparticles: journey inside the cell. Chem. Soc. Rev. 46 4218-4244 (2017)

161. Trimble, W. S. \& Grinstein, S. Barriers to the free diffusion of proteins and lipids in the plasma membrane. J. Cell Biol. 208, 259-271 (2015)

162. Foroozandeh, P. \& Aziz, A. A. Insight into cellular uptake and intracellular trafficking of nanoparticles. Nanoscale Res. Lett. 13, 339 (2018).

163. Zhang, L. et al. Shape effect of nanoparticles on tumor penetration in monolayers versus spheroids. Mol. Pharm. 16, 2902-2911 (2019).

164. Lunnoo, T., Assawakhajornsak, J. \& Puangmali, T. In silico study of gold nanoparticle uptake into a mammalian cell: interplay of size, shape, surface charge, and aggregation. J. Phys. Chem. C. 123 3801-3810 (2019)

165. Zhang L et al. Tumor chemo-radiotherapy with rod-shaped and spherical gold nano probes: shape and active targeting both matter. Theranostics 9 , 1893-1908 (2019)

166. Dzuricky, M., Xiong, S., Weber, P. \& Chilkoti, A. Avidity and cell uptake of integrin-targeting polypeptide micelles is strongly shape-dependent. Nano Lett. 19, 6124-6132 (2019).

167. Shang, L., Nienhaus, K. \& Nienhaus, G. U. Engineered nanoparticles interacting with cells: size matters. J. Nanobiotechnol. 12, 5 (2014).

168. Zhang, L., Chen, H., Xie, J., Becton, M. \& Wang, X Interplay of nanoparticle rigidity and its translocation ability through cell membrane. J. Phys. Chem. B 123 , 8923-8930 (2019).
169. Hocking, K. M. et al. Nanotechnology enabled modulation of signaling pathways affects physiologic responses in intact vascular tissue. Tissue Eng. Part $A$ 25, 416-426 (2019)

170. Shahin, M., Safaei-Nikouei, N. \& Lavasanifar, A. Polymeric micelles for $\mathrm{pH}$-responsive delivery of cisplatin. J. Drug Target 22, 629-637 (2014).

171. Chen, L. Q. et al. Nanotoxicity of silver nanoparticles to red blood cells: size dependent adsorption, uptake, and hemolytic activity. Chem. Res. Toxicol. 28, 501-509 (2015).

172. Yue, J., Feliciano, T. J., Li, W., Lee, A. \& Odom, T. W. Gold nanoparticle size and shape effects on cellular uptake and intracellular distribution of siRNA nanoconstructs. Bioconjug. Chem. 28, 1791-1800 (2017).

173. Chen, G. et al. A biodegradable nanocapsule delivers a Cas9 ribonucleoprotein complex for in vivo genome editing. Nat. Nanotechnol. 14, 974-980 (2019).

174. Miao, L. et al. Delivery of mRNA vaccines with heterocyclic lipids increases anti-tumor efficacy by STING-mediated immune cell activation. Nat. Biotechnol. 37, 1174-1185 (2019).

175. Maugeri, M. et al. Linkage between endosomal escap of LNP-mRNA and loading into EVs for transport to other cells. Nat. Commun. 10, 4333 (2019).

176. Almeida, A. P. B. et al. Mucopenetrating lipoplexes modified with PEG and hyaluronic acid for CD44targeted local siRNA delivery to the lungs. J. Biomater. Appl. 34, 617-630 (2019).

177. Whitehead, K. A. et al. Degradable lipid nanoparticles with predictable in vivo siRNA delivery activity. Nat. Commun. 5, 4277 (2014).

178. Oberli, M. A. et al. Lipid nanoparticle assisted mRNA delivery for potent cancer immunotherapy. Nano Lett. 17, 1326-1335 (2017).

179. Kaczmarek, J. C. et al. Optimization of a degradable polymer-lipid nanoparticle for potent systemic delivery of mRNA to the lung endothelium and immune cells. Nano Lett. 18, 6449-6454 (2018).

180. Wei, L., Zhao, Y., Hu, X. \& Tang, L. Redox-responsive polycondensate neoepitope for enhanced personalized cancer vaccine. ACS Cent. Sci. 6, 404-412 (2020).

181. Gao, Y. et al. $\mathrm{pH} /$ redox dual-responsive polyplex with effective endosomal escape for codelivery of siRNA and doxorubicin against drug-resistant cancer cells. ACS Appl. Mater. Interfaces 11, 16296-16310 (2019).

182. Yuan, P. et al. Mitochondria-targeting, intracellular delivery of native proteins using biodegradable silica nanoparticles. Angew. Chem. 131, 7739-7743 (2019).

183. Yamada, Y., Fukuda, Y., Sasaki, D., Maruyama, M $\&$ Harashima, H. Development of a nanoparticle that releases nucleic acids in response to a mitochondrial environment. Mitochondrion 52, 67-74 (2020).

184. Foroozandeh, P., Aziz, A. A. \& Mahmoudi, M. Effect of cell age on uptake and toxicity of nanoparticles: the overlooked factor at the nanobio interface. ACS Appl. Mater. Interfaces 11, 39672-39687 (2019).

185. Serpooshan, V. et al. Effect of cell sex on uptake of nanoparticles: the overlooked factor at the nanobio interface. ACS Nano 12, 2253-2266 (2018)

186. Dong, Y. et al. Targeted blocking of miR328 lysosomal degradation with alkalized exosomes sensitizes the chronic leukemia cells to imatinib. Appl. Microbiol. Biotechnol. 103, 9569-9582 (2019).

187. Yang, X. et al. Delivery of platinum (II) drugs with bulky ligands in trans-geometry for overcoming cisplatin drug resistance. Mater. Sci. Eng. C. 96, 96-104 (2019).

188. Murakami, M. et al. Improving drug potency and efficacy by nanocarrier-mediated subcellular targeting. Sci. Transl. Med. 3, 64ra2 (2011).

189. American Cancer Society. Cancer facts \& figures 2019 (ACS, 2019).

190. Dalby, M., Cree, I. A., Challoner, B. R., Ghosh, S $\&$ Thurston, D. E. The precision medicine approach to cancer therapy: part 1 - solid tumours. Pharm. J. https://doi.org/10.1211/PJ.2019.20207119 (2019)

191. Hauschild, A. et al. Long-term outcomes in patients with BRAF V600-mutant metastatic melanoma receiving dabrafenib monotherapy: analysis from phase 2 and 3 clinical trials. Eur. J. Cancer 125 114-120 (2020)

192. Chapman, P. B. et al. Improved survival with vemurafenib in melanoma with BRAF V600E mutation. N. Engl. J. Med. 364, 2507-2516 (2011).

193. Ou, W. et al. Regulatory T cell-targeted hybrid nanoparticles combined with immuno-checkpoint blockage for cancer immunotherapy. J. Control. Rel. 281, 84-96 (2018).
194. Lyon, R. P. et al. Self-hydrolyzing maleimides improve the stability and pharmacological properties of antibody-drug conjugates. Nat. Biotechnol. 32, 1059-1062 (2014).

195. Roma-Rodrigues, C., Mendes, R., Baptista, P. V. $\&$ Fernandes, A. R. Targeting tumor microenvironment for cancer therapy. Int. J. Mol. Sci. 20, 840 (2019).

196. Rao, L. et al. Cancer cell membrane-coated nanoparticles for personalized therapy in patientderived xenograft models. Adv. Funct. Mater. 29 1905671 (2019)

197. He, Z., Zhang, Y. \& Feng, N. Cell membrane-coated nanosized active targeted drug delivery systems homing to tumor cells: a review. Mater. Sci. Eng. C. 106, 110298 (2020).

198. Wang, Y., Luan, Z., Zhao, C., Bai, C. \& Yang, K. Target delivery selective CSF-1R inhibitor to tumor-associated macrophages via erythrocyte-cancer cell hybrid membrane camouflaged pH-responsive copolymer micelle for cancer immunotherapy. Eur. J. Pharm. Sci. 142, 105136 (2020).

199. Li, R., He, Y., Zhang, S., Qin, J. \& Wang, J. Cell membrane-based nanoparticles: a new biomimetic platform for tumor diagnosis and treatment. Acta Pharm. Sin. B 8, 14-22 (2018).

200. Karlsson, J. et al. Engineered nanoparticles for systemic siRNA delivery to malignant brain tumours. Nanoscale 11, 20045-20057 (2019).

201. Ramchandani, D. et al. Nanoparticle delivery of miR708 mimetic impairs breast cancer metastasis. Mol. Cancer Ther. 18, 579-591 (2019).

202. Zhang, Y. R. et al. Strategies to improve tumor penetration of nanomedicines through nanoparticle design. Wiley Interdiscip. Rev. Nanomed. Nanobiotechnol. 11, 1-12 (2019).

203. Xu, C. et al. Biodegradable nanotheranostics with hyperthermia-induced bubble ability for ultrasound imaging-guided chemo-photothermal therapy. Int. J. Nanomed. 14, 7141-7153 (2019).

204. Li, H. J. et al. Stimuli-responsive clustered nanoparticles for improved tumor penetration and therapeutic efficacy. Proc. Natl Acad. Sci. USA 113 4164-4169 (2016).

205. Wang, D. et al. Engineering nanoparticles to locally activate T cells in the tumor microenvironment. Sci. Immunol. 4, 1-14 (2019).

206. Kim, C. S., Duncan, B., Creran, B. \& Rotello, V. M. Triggered nanoparticles as therapeutics. Nano Today 8, 439-447 (2013).

207. Wong, C. et al. Multistage nanoparticle delivery system for deep penetration into tumor tissue. Proc. Natl Acad. Sci. USA 108, 2426-2431 (2011).

208. Lim, W. Q., Phua, S. Z. F. \& Zhao, Y. Redox-responsive polymeric nanocomplex for delivery of cytotoxic protein and chemotherapeutics. ACS Appl. Mater. Interfaces 11, 31638-31648 (2019).

209. Mannaris, C. et al. Acoustically responsive polydopamine nanodroplets: a novel theranostic agent. Ultrason. Sonochem. 60, 104782 (2020).

210. Riley, R. S. et al. Evaluating the mechanisms of light-triggered siRNA release from nanoshells for temporal control over gene regulation. Nano Lett. 18 3565-3570 (2018)

211. Dariva, C. G., Coelho, J. F. J. \& Serra, A. C. Near infrared light-triggered nanoparticles using singlet oxygen photocleavage for drug delivery systems. J. Control. Rel. 294, 337-354 (2019).

212. Shi, H. et al. Programmed co-delivery of platinum nanodrugs and gemcitabine by a clustered nanocarrier for precision chemotherapy for NSCLC tumors. J. Mater. Chem. B 8, 332-342 (2020).

213. Li, H. J. et al. Smart superstructures with ultrahigh $\mathrm{pH}$-sensitivity for targeting acidic tumor microenvironment: instantaneous size switching and improved tumor penetration. ACS Nano 10 , 6753-6761 (2016).

This study demonstrates the usage of $\mathrm{pH}$ triggered particle size-switching in the tumour microenvironment to facilitate NP extravasation and accumulation.

214. Chen, W. H., Luo, G. F. \& Zhang, X. Z. Recent advances in subcellular targeted cancer therap based on functional materials. Adv. Mater. 31, 1-39 (2019).

215. Goswami, U. et al. Transferrin-copper nanoclusterdoxorubicin nanoparticles as targeted theranostic cancer nanodrug. ACS Appl. Mater. Interfaces 10 3282-3294 (2018).

216. Qiao, Y. et al. Stimuli-responsive nanotherapeutics for precision drug delivery and cancer therapy. Wiley Interdiscip. Rev. Nanomed. Nanobiotechnol. 11, 1-20 (2019). 
217. Jin, Q., Deng, Y., Chen, X. \& Ji, J. Rational design of cancer nanomedicine for simultaneous stealth surface and enhanced cellular uptake. ACS Nano 13 954-977 (2019)

218. Fang, Y. et al. Cleavable PEGylation: a strategy for overcoming the "PEC dilemma" in efficient drug delivery. Drug Deliv. 24, 22-32 (2017).

219. Guo, F. et al. Matrix metalloprotein-triggered, cell penetrating peptide-modified star-shaped nanoparticles for tumor targeting and cancer therapy. J. Nanobiotechnol. 18, 48 (2020).

220. Eno, J. Immunotherapy through the years. J. Adv. Pract. Oncol. 8, 747-753 (2017).

221. Marshall, H. T. \& Djamgoz, M. B. A. Immuno-oncology: emerging targets and combination therapies. Front. Oncol. 8, 1-29 (2018).

222. Urbanavicius, D. Alvarez, T., Such, G. K Johnston, A. P. R. \& Mintern, J. D. The potential of nanoparticle vaccines as a treatment for cancer. Mol. Immunol. 98, 2-7 (2018).

223. Truex, N. L. et al. Automated flow synthesis of tumor neoantigen peptides for personalized immunotherapy. Sci. Rep. 10, 723 (2020).

224. Guo, Y., Lei, K. \& Tang, L. Neoantigen vaccine delivery for personalized anticancer immunotherapy. Front Immunol. 9, 1499 (2018).

225. Dombroski, J. A., Jyotsana, N., Crews, D. W., Zhang, Z. \& King, M. R. Fabrication and characterization of tumor nano-lysate as a preventative vaccine for breast cancer. Langmuir 36, 6531-6539 (2020).

226. Gu, P. et al. Rational design of PLGA nanoparticle vaccine delivery systems to improve immune responses. Mol. Pharm. 16, 5000-5012 (2019).

227. Firdessa-Fite, R. \& Creusot, R. J. Nanoparticles versus dendritic cells as vehicles to deliver mRNA encoding multiple epitopes for immunotherapy. Mol. Ther Methods Clin. Dev. 16, 50-62 (2019).

228. Soema, P. C., Willems, G. J., Jiskoot, W., Amorij, J. P. \& Kersten, G. F. Predicting the influence of liposomal lipid composition on liposome size, zeta potential and liposome-induced dendritic cell maturation using a design of experiments approach. Eur. J. Pharm. Biopharm. 94, 427-435 (2015)

229. Cao, F., Yan, M., Liu, Y., Liu, L. \& Ma, G. Photothermally controlled MHC class I restricted CD8+ T-cell responses elicited by hyaluronic acid decorated gold nanoparticles as a vaccine for cancer immunotherapy. Adv. Healthc. Mater. 7, 1-12 (2018)

230. An, M., Li, M., Xi, J. \& Liu, H. Silica nanoparticle as a lymph node targeting platform for vaccine delivery. ACS Appl. Mater. Interfaces 9, 23466-23475 (2017).

231. Fontana, F., Liu, D., Hirvonen, J. \& Santos, H. A Delivery of therapeutics with nanoparticles: what's new in cancer immunotherapy? Wiley Interdiscip. Rev. Nanomed. Nanobiotechnol. 9, 1-26 (2017).

232. Tran, T. H. et al. Nanoparticles for dendritic cell-based immunotherapy. Int. J. Pharm. 542, 253-265 (2018).

233. Grippin, A. J., Sayour, E. J. \& Mitchell, D. A Translational nanoparticle engineering for cancer vaccines. Oncoimmunology 6, e1 290036 (2017).

234. Liu, J., Miao, L., Sui, J., Hao, Y. \& Huang, G. Nanoparticle cancer vaccines: design considerations and recent advances. Asian J. Pharm. Sci. 15 576-590 (2019)

235. Tostanoski, L. H., Gosselin, E. A. \& Jewell, C. M. Engineering tolerance using biomaterials to target and control antigen presenting cells. Discov. Med. 21 403-410 (2016).

236. Zupan\&\#x010D;i\&\#x010D;, E. et al. Rational design of nanoparticles towards targeting antigen-presenting cells and improved T cell priming. J. Control. Rel. 258, 182-195 (2017)

237. Macri, C., Dumont, C., Johnston, A. P. R. \& Mintern, J. D. Targeting dendritic cells: a promising strategy to improve vaccine effectiveness. Clin. Transl. Immunol. 5, e66-e68 (2016).

238. Zhang, F. et al. Genetic programming of macrophages to perform anti-tumor functions using targeted mRNA nanocarriers. Nat. Commun. 10, 3974 (2019).

239. Li, K. et al. Polarization of tumor-associated macrophage phenotype via porous hollow iron nanoparticles for tumor immunotherapy in vivo. Nanoscale 12, 130-144 (2020)

240. Chavez-Santoscoy, A. V. et al. Tailoring the immune response by targeting $C$-type lectin receptors on alveolar macrophages using 'pathogen-like' amphiphilic polyanhydride nanoparticles. Biomaterials 33, 4762-4772 (2012)

241. Zhang, C. et al. Targeted antigen delivery to dendritic cell via functionalized alginate nanoparticles for cancer immunotherapy. J. Control Release 256, 170-181 (2017).

242. Chen, W. C. et al. Antigen delivery to macrophages using liposomal nanoparticles targeting sialoadhesin/ CD169. PLoS One 7, 1-9 (2012).

243. Tatar, A. S. et al. CD19-targeted, Raman tagged gold nanourchins as theranostic agents against acute lymphoblastic leukemia. Colloids Surf. B Biointerfaces 184, 110478 (2019).

244. Qian, Y. et al. Targeting dendritic cells in lymph node with an antigen peptide-based nanovaccine for cancer immunotherapy. Biomaterials 98, 171-183 (2016).

245. Pearson, R. M., Casey, L. M., Hughes, K. R., Miller, S. D. $\&$ Shea, L. D. In vivo reprogramming of immune cells: technologies for induction of antigen-specific tolerance. Adv. Drug Deliv. Rev. 114, 240-255 (2017).

246. Singh, A. \& Peppas, N. A. Hydrogels and scaffolds for immunomodulation. Adv. Mater. 26, 6530-654 (2014).

247. Marloye, M., Lawler, S. E. \& Berger, G. Current patent and clinical status of stimulator of interferon genes (STING) agonists for cancer immunotherapy. Pharm Pat. Anal. 8, 87-90 (2019).

248. Liu, Y. et al. An inhalable nanoparticulate STING agonist synergizes with radiotherapy to confer long term control of lung metastases. Nat. Commun. 10 $1-15$ (2019)

249. Cheng, N. et al. A nanoparticle-incorporated STING activator enhances antitumor immunity in PDL1-insensitive models of triple-negative breast cancer JCl Insight 3, 1-20 (2018)

250. Wilson, D. R. et al. Biodegradable STING agonist nanoparticles for enhanced cancer immunotherapy. Nanomed. Nanotechnol. Biol. Med. 14, 237-246 (2018).

251. Schmid, D. et al. T cell-targeting nanoparticles focus delivery of immunotherapy to improve antitumor immunity. Nat. Commun. 8, 1-11 (2017).

252. Wang, C., Ye, Y., Hochu, G. M., Sadeghifar, H. \& Gu, Z. Enhanced cancer immunotherapy by microneedle patch-assisted delivery of anti-PD1 antibody. Nano Lett. 16, 2334-2340 (2016)

253. Wang, C., Sun, W., Wright, G., Wang, A. Z. \& Gu, Z. Inflammation-triggered cancer immunotherapy by programmed delivery of $\mathrm{CpG}$ and Anti-PD1 antibody. Adv. Mater. 28, 8912-8920 (2016).

254. Deng, H. \& Zhang, Z. The application of nanotechnology in immune checkpoint blockade for cancer treatment. J. Control. Rel. 290, 28-45 (2018).

255. Dunn, Z. S., Mac, J. \& Wang, P. T cell immunotherapy enhanced by designer biomaterials. Biomaterials 217 119265 (2019).

256. Hickey, J. W., Vicente, F. P., Howard, G. P., Mao, H. Q. \& Schneck, J. P. Biologically inspired design of nanoparticle artificial antigen-presenting cells for immunomodulation. Nano Lett. 17, 7045-7054 (2017).

257. Sunshine, J. C., Perica, K., Schneck, J. P. \& Green, J. J. Particle shape dependence of $C D 8^{+} T$ cell activation by artificial antigen presenting cells. Biomaterials 35 , 269-277 (2014)

258. Billingsley, M. et al. lonizable lipid nanoparticle mediated mRNA delivery for human CAR T cell engineering. Nano Lett. 20, 1578-1589 (2020).

259. Smith, T. T. et al. In situ programming of leukaemiaspecific $T$ cells using synthetic DNA nanocarriers. Nat. Nanotechnol. 12, 813-822 (2017).

260. Pozsgay, J., Szekanecz, Z. \& Sármay, G. Antigenspecific immunotherapies in rheumatic diseases. Nat. Rev. Rheumatol. 13, 525-537 (2017).

261. Jung, H. H. et al. Polymeric nanoparticles containing both antigen and vitamin D3 induce antigen-specific immune suppression. Immune Netw. 19, 1-12 (2019).

262. Pang, L., Macauley, M. S., Arlian, B. M., Nycholat, C. M. \& Paulson, J. C. Encapsulating an immunosuppressant enhances tolerance induction by Siglec-engaging tolerogenic liposomes. ChemBioChem 18, 1226-1233 (2017)

263. Horwitz, D. A., Bickerton, S., Koss, M., Fahmy, T. M. \& La Cava, A Suppression of murine lupus by CD4 and $C D 8^{+} T_{\text {reg }}$ cells induced by $T$ cell-targeted nanoparticles loaded with interleukin- 2 and transforming growth factor $\beta$. Arthritis Rheumatol. 71 632-640 (2019)

264. Hu, T. et al. pVAX1-A20 alleviates colitis in mice by promoting regulatory T cells. Dig. Liver Dis. $\mathbf{5 1}$ 790-797 (2019)

265. Shahzad, K. A. et al. Design and optimization of PLGA particles to deliver immunomodulatory drugs for the prevention of skin allograft rejection. Immunol. Invest. 49, 840-857 (2019).

266. Li, R. et al. Sustained release of immunosuppressant by nanoparticle-anchoring hydrogel scaffold improved the survival of transplanted stem cells and tissue regeneration. Theranostics 8, 878-893 (2018).

267. Zhang, Y. et al. In situ repurposing of dendritic cells with CRISPR/Cas9-based nanomedicine to induce transplant tolerance. Biomaterials 217, 119302 (2019).

268. Yin, H., Kauffman, K. J. \& Anderson, D. G. Delivery technologies for genome editing. Nat. Rev. Drug Discov. 16, 387-399 (2017).

269. Yin, H. et al. Non-viral vectors for gene-based therapy. Nat. Rev. Genet. 15, 541-555 (2014).

270. Riley, R. S., June, C. H., Langer, R. \& Mitchell, M. J. Delivery technologies for cancer immunotherapy. Nat. Rev. Drug Discov. 18, 175-196 (2019).

271. Cavazzana-Calvo, M. et al. Gene therapy of human severe combined immunodeficiency (SCID)-X1 disease. Science 288, 669-672 (2000).

272. Hacein-Bey-Abina, S et al. LMO2-associated clonal $\mathrm{T}$ cell proliferation in two patients after gene therapy for SCID-X1. Science 302, 1181-1185 (2003).

273. Whitehead, K. A., Langer, R. \& Anderson, D. G. Knocking down barriers: advances in siRNA delivery. Nat. Rev. Drug Discov. 8, 129-138 (2009).

274. Semple, S. C. et al. Rational design of cationic lipids for siRNA delivery. Nat. Biotechnol. 28, 172-176 (2010).

275. Davis, M. E. et al. Evidence of RNAi in humans from systemically administered siRNA via targeted nanoparticles. Nature 464, 1067-1070 (2010).

276. Mangraviti, A. et al. Polymeric nanoparticles for nonviral gene therapy extend brain tumor survival in vivo. ACS Nano 9, 1236-1249 (2015).

277. Sarett, S. M. et al. Lipophilic siRNA targets albumin in situ and promotes bioavailability, tumor penetration, and carrier-free gene silencing. Proc. Natl Acad. Sci. USA 114, E6490-E6497 (2017).

278. He, Y. et al. Polyethyleneimine/DNA polyplexes with reduction-sensitive hyaluronic acid derivatives shielding for targeted gene delivery. Biomaterials 34 , 1235-1245 (2013).

279. Xu, C. F. et al. Rational designs of in vivo CRISPR-Cas delivery systems. Adv. Drug Deliv. Rev. https://doi.org/ 10.1016/j.addr.2019.11.005 (2019).

280. Maule, G. et al. Allele specific repair of splicing mutations in cystic fibrosis through AsCas 12a genome editing. Nat. Commun. 10, 3556 (2019).

281. Moss, R. B. et al. Repeated aerosolized AAV-CFTR for treatment of cystic fibrosis: a randomized placebo-controlled phase 2B trial. Hum. Gene Ther. 18, 726-732 (2007)

282. Alton, E. W. F. W. et al. Repeated nebulisation of nonviral CFTR gene therapy in patients with cystic fibrosis a randomised, double-blind, placebo-controlled, phase 2b trial. Lancet Respir. Med. 3, 684-691 (2015).

283. Guan, S. et al. Self-assembled peptide-poloxamine nanoparticles enable in vitro and in vivo genome restoration for cystic fibrosis. Nat. Nanotechnol. 14 287-297 (2019).

284. Robinson, E. et al. Lipid nanoparticle-delivered chemically modified mRNA restores chloride secretion in cystic fibrosis. Mol. Ther. 26, 2034-2046 (2018).

285. Witten, J., Samad, T. \& Ribbeck, K. Selective permeability of mucus barriers. Curr. Opin. Biotechnol. 52, 124-133 (2018).

286. Patel, A. K. et al. Inhaled nanoformulated mRNA polyplexes for protein production in lung epithelium. Adv. Mater. 31, 1805116 (2019)

287 . Suk, J. S. et al. Lung gene therapy with highly compacted DNA nanoparticles that overcome the mucus barrier. J. Control. Rel. 178, 8-17 (2014).

288. Deweerdt, S. The fix is in utero. Nature $564,6-8$ (2018).

289. Massaro, G. et al. Fetal gene therapy for neurodegenerative disease of infants. Nat. Med. 24, 1317-1323 (2018).

290. Ricciardi, A. S. et al. In utero nanoparticle delivery for site-specific genome editing. Nat. Commun. 9, 1-11 (2018).

291. Caster, J. M., Patel, A. N., Zhang, T. \& Wang, A Investigational nanomedicines in 2016: a review of nanotherapeutics currently undergoing clinical trials. Wiley Interdiscip. Rev. Nanomed. Nanobiotechnol. 9, 1416 (2017).

292. Wu, S. et al. Rapid label-free isolation of circulating tumor cells from patients' peripheral blood using electrically charged $\mathrm{Fe}_{3} \mathrm{O}_{4}$ nanoparticles. ACS Appl. Mater. Interfaces 12, 4193-4203 (2020). 


\section{REVIEWS}

293. León-Janampa, N. et al. Synthesis, characterization and bio-functionalization of magnetic nanoparticles to improve the diagnosis of tuberculosis.

Nanotechnology 31, 175101 (2020).

294. Muhammad, P. et al. SERS-based nanostrategy for rapid anemia diagnosis. Nanoscale 12, 1948-1957 (2020).

\section{Acknowledgements}

M.J.M. acknowledges support from a Burroughs Wellcome Fund Career Award at the Scientific Interface (CASI), a US National Institutes of Health (NIH) Director's New Innovator Award (DP2 TR002776), a grant from the American Cance Society (1 29784-IRG-16-188-38-IRG), the NIH (NCI R01 CA241661, NCI R37 CA244911 and NIDDK R01 DK1 23049), an Abramson Cancer Center (ACC)-School o Engineering and Applied Sciences (SEAS) Discovery Grant (P30 CA016520) and a 2018 American Association for Cancer
Research (AACR)-Bayer Innovation and Discovery Grant (Grant Number 18-80-44-MITC) N.A.P acknowledges support from the UT-Portugal Collaborative Research Program (CoLAB), the NIH (R01-EB022025-4 and R01-EB-00246-21), the National Science Foundation (Grant 1033746), the Pratt Foundation, the Cockrell Family Regents Chair and NSF Graduate Research Fellowships. R.M.H. was supported by a National Science Foundation (NSF) Graduate Research Fellowship (DGE 1845298). M.M.B. was supported by an NIH Training in HIV Pathogenesis T32 Program (T32 AI007632).

\section{Author contributions}

M.M.B. and R.M.H. conducted the initial literature search and outlined the general manuscript format. M.M.B. R.M.H. and M.E.W. wrote the initial manuscript draft with contributions from M.J.M., N.A.P. and R.L. All authors reviewed and critically revised previous versions of the manuscript. All authors read and approved the final manuscripts.

\section{Competing interests}

A list of entities with which R.L. is involved, compensated or uncompensated, can be found in the Supplementary information. M.J.M., M.M.B., R.M.H., M.E.W. and N.A.P. declare no competing interests.

\section{Publisher's note}

Springer Nature remains neutral with regard to jurisdictional claims in published maps and institutional affiliations.

Supplementary information

Supplementary information is available for this paper at https://doi.org/10.1038/s41573-020-0090-8.

(c) Springer Nature Limited 2020 